\title{
Polarization properties of broad absorption line QSOs: New statistical clues $\star, \star \star$
}

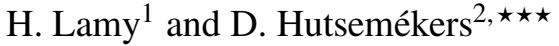 \\ ${ }^{1}$ IASB - BIRA, Avenue Circulaire 3, 1180 Bruxelles, Belgium \\ e-mail: herve.lamy@oma.be \\ 2 Institut d'Astrophysique, Université de Liège, Sart-Tilman, 4000 Liège, Belgium
}

Received 9 April 2004 / Accepted 19 July 2004

\begin{abstract}
We report the results of several statistical tests performed on a large sample of 139 broad absorption line (BAL) QSOs with good quality optical spectra and/or optical polarization data. Correlations between ten optical indices and the polarization degree $p_{0}$ are systematically searched for. We find six significant non-trivial correlations. In order to identify the most important correlations, we perform a principal component analysis with a sample of 30 BAL QSOs and eight quantities (including $p_{0}$ ). Most of the variance $(\sim 57 \%)$ in the data is contained in two principal components called PC1 and PC2. PC1 is mainly dominated by the correlation between the balnicity index BI and the strength of the Fe II emission; it may be driven by the accretion rate of matter onto the central compact object. The variance in PC2 is essentially due to the anti-correlation between $p_{0}$ and the detachment index DI, indicating that BAL QSOs with P Cygni profiles (DI $\ll$ ) are usually more polarized than those objects with C IV absorption troughs well detached from the corresponding emission lines (DI»). We show that PC2 may be related to the orientation of the BAL QSOs with respect to the line of sight.

We also present new spectropolarimetric observations of six BAL QSOs. By adding spectropolarimetric data from the literature, we build a sample of 21 BAL QSOs for which we define four spectropolarimetric indices describing the polarization properties of the absorption and emission lines. We find that the polarization of the $\mathrm{C} \mathrm{III]} \mathrm{emission} \mathrm{line} \mathrm{is} \mathrm{systematically} \mathrm{higher} \mathrm{than} \mathrm{the} \mathrm{po-}$ larization of the C IV emission line, and that the highest polarization in the troughs is correlated to the balnicity index. Another important result emerging from the statistical tests performed on this spectropolarimetric sample is a possible anti-correlation between the detachment index and a quantity SI which measures the ratio of the depths of the CIV absorption in the polarized flux and in the total flux. This correlation indicates that in BAL QSOs with P cygni profiles, the BAL troughs in the polarized flux are nearly as deep as in the total flux while, in BAL QSOs with detached absorptions, the BAL troughs in the polarized flux are much weaker than in the total flux.

We show that our main results may be explained in the framework of a "two-component" wind model which is a natural extension of the classical wind-from-disk models. In this model, the broad absorption occurs in a dense equatorial wind emerging from the accretion disk, while scattering and polarization mainly take place in a polar region. The orientation relative to the observer drives the correlations $p_{0}-$ DI and DI - SI. While most of our observations can be explained within this framework, there are also several indications that other polarization mechanisms, and more particularly resonance scattering, may also be at work.
\end{abstract}

Key words. galaxies: quasars: general - galaxies: quasars: absorption lines - polarization

\section{Introduction}

About 10 to $20 \%$ of optically-selected QSOs exhibit broad absorption lines (BAL) in the resonance lines of highly ionized species such as N v $\lambda 1240$, C IV $\lambda 1549$ or Si IV $\lambda 1397$ (Hewett \& Foltz 2003; Tolea et al. 2002; Reichard et al. 2003).

\footnotetext{
^ Based on observations collected at the European Southern Observatory (ESO, La Silla).

$\star \star$ Table 1 is only available in electronic form at the CDS via anonymous ftp to cdsarc.u-strasbg.fr $(130.79 .128 .5)$ or via http://cdsweb.u-strasbg.fr/cgi-bin/qcat?]/A+A/427/107 $\star \star \star$ Also, Chercheur Qualifié au Fonds National de la Recherche Scientifique (FNRS, Belgium).
}

A smaller fraction $(\sim 1 \%)$ has in addition broad absorption lines from lower ionization species like $\mathrm{Mg}$ II $\lambda 2798$ or $\mathrm{Al}$ III $\lambda 1858$. These objects are often referred to as LoBAL or LIBAL QSOs in the literature, in contrast with those objects with only high ionization BALs, hereafter denoted HIBAL QSOs ${ }^{1}$. The broad absorption lines are blueshifted relative to the emission lines, indicating the presence of outflows with velocities ranging up to $\sim 0.2 c$.

\footnotetext{
${ }^{1}$ In order to be consistent with the notations introduced in our previous papers, we use here the acronyms HIBAL-LIBAL QSO instead of the more widely used HiBAL-LoBAL QSO dichotomy. The differences are explained in Sect. 2.2.
} 
Despite of more than three decades of intense observation and modelling, the fundamental nature of the BAL QSOs remains controversial. Indeed, the physical mechanism that drives the flow is still a matter of debate although there are some indications that the outflows are driven by radiation pressure on spectral lines and originate from an accretion disk rotating around a massive black hole (Murray et al. 1995; Arav 1997; Proga et al. 2000). Acceleration of the outflow by other mechanisms such as gas pressure (Begelman et al. 1991), magnetic fields (de Kool \& Begelman 1995) or radiation pressure by dust (Voit et al. 1993; Scoville \& Norman 1995; Everett et al. 2002) has also been proposed and may contribute as well. Moreover, it is not yet understood whether BAL QSOs and non-BAL QSOs are completely different types of quasars or if their properties mainly depend on their orientation towards us.

In order to determine if BAL QSOs and non-BAL QSOs are drawn from the same parent population of quasars, Weymann et al. (1991, hereafter W91) carried out a detailed comparative study of the emission lines and continuum properties of a sample of 42 BAL QSOs and 29 non-BAL QSOs. Recently, Reichard et al. (2003) made a similar analysis with a much larger sample (224 BAL QSOs and 892 non-BAL QSOs) drawn from the SDSS EDR (Sloan Digital Sky Survey Early Data Release, Stoughton et al. 2002), which essentially confirms the results of W91: there are no significant differences between emission line properties of BAL QSOs and non-BAL QSOs, except a noticeable enhancement of $\mathrm{N} \mathrm{V}$ emission in BAL QSOs. Also, the continua of the BAL QSOs are redder (Reichard et al. 2003), especially for those objects with low ionization absorption lines. This is possibly due to a larger dust content (Sprayberry \& Foltz 1992; Yamamoto \& Vansevicius 1999). Both statistical studies lead to conclusion that BAL QSOs and non-BAL QSOs come from a single population. The small differences observed in their emission line/continuum properties may indicate that BAL QSOs are normal QSOs with extreme properties such as larger accretion rates (Reichard et al. 2003; Yuan \& Wills 2003).

Partly based on these results, the most popular paradigm considers that a broad absorption line region (BALR) exists in all QSOs and has a covering factor $\sim 0.1$, i.e. close to the rate of detection of BAL QSOs among optically-selected QSOs. In this orientation scheme, broad absorption lines are observed only when our line of sight intercepts the outflow. A completely different possibility is to consider that the BAL phenomenon is an evolutionary phase with significant mass loss during the life of a QSO (Voit et al. 1993). The best argument supporting this evolutionary model is given by the large percentage of LIBAL QSOs detected among infrared surveys (Low et al. 1989), suggesting that these objects are enshrouded in a cocoon of dust which heavily attenuates the optical/UV continuum (Sprayberry \& Foltz 1992). Moreover, in order to explain the very weak $[\mathrm{O}$ III] intensity measured in those LIBAL QSOs (Boroson \& Green 1992; Yuan \& Wills 2003), the covering factor of the BALR must be much larger than 0.1.

Since we want to test for orientation as a possible keyparameter to understand the BAL QSO/non-BAL QSO dichotomy, polarization may be a useful tool because it is sensitive to the geometry and orientation of these unresolved objects. Previous optical polarization surveys have shown that, as a class, BAL QSOs are more polarized than non-BAL QSOs and that the polarization is most probably due to scattering (Moore \& Stockman 1984; Hutsemékers et al. 1998, hereafter Paper I; Schmidt \& Hines 1999; Hutsemékers \& Lamy 2002; Lamy 2003). The fact that BAL QSOs are polarized reveals that the scattering regions are not spherically symmetric. Many current theoretical models of BAL QSOs assume an axisymmetric geometry, with either an accretion disk (e.g. Murray et al. 1995) or an opaque dusty torus (e.g. Schmidt \& Hines 1997) located in the equatorial plane. In this equatorial geometry, the BALR emerges either from the accretion disk in the form of a continuous flow or it is ablated from the top of the dusty torus in the form of dense cloudlets. It is then accelerated to large velocities. However, there exists recent models which locate the BALR within a weak jet in the polar regions in order to explain the radio-quiet/radio-loud dichotomy and to simultaneously relate the BAL phenomenom to the associated absorptions often observed in radio-loud QSOs (Punsly 1999; Kuncic 1999). In this polar geometry, the BALR is embedded in a poorly collimated radio jet and is accelerated by the pressure of the surrounding medium which also acts to confine the BAL clouds.

Spectropolarimetry has been used to put constrains on the structure of BAL QSOs (Glenn et al. 1994; Cohen et al. 1995; Goodrich \& Miller 1995; Schmidt \& Hines 1999; Ogle et al. 1999). It has revealed the presence of a polarized component filling in the absorption troughs and attributed to a continuum component polarized by scattering off electrons or dust. The scattered and direct rays suffer different amounts of absorption which results in a strong increase of the polarization in the BAL troughs. Spectropolarimetry has also been used to determine the relative size of the different emission/absorption/scattering regions in BAL QSOS. However, several axisymetric configurations are possible to interpret the spectropolarimetric observations (Ogle 1997). Therefore, we have to find other parameters which, in connection to the polarization data, may help to discriminate among the different models of BAL QSOs.

In this perspective, we performed in Paper I a systematic search of correlations between optical broad-band polarization and several indices characterizing the optical spectra of BAL QSOs in order to have a clue on the nature of the outflows. We find that the main correlations involving $p_{0}$ could be naturally interpreted within the "wind-from-disk" (hereafter WfD) model of Murray et al. (1995). However, the size of the sample was small (29 objects) and some important but statistically marginal trends noticed in Paper I need to be confirmed. Schmidt \& Hines (1999) also report on a marginal correlation between $p_{0}$ and the strength of the C IV broad absorption lines, which was not detected in Paper I. Since then, a lot of good quality polarimetric data of BAL QSOs have been obtained (Lamy \& Hutsemékers 2000a; Sluse et al. 2004; Hutsemékers et al. 2004) and the existence of a population of radio loud QSOs with intrinsic, BAL-like outflows has been confirmed (Becker et al. 2000, 2001; Menou et al. 2001). It is therefore important to perform these statistical tests again using a much larger sample of BAL QSOs than in Paper I (139 objects instead of 29), in order to better identify which spectral characteristics of BAL QSOs are orientation dependent. This is 
the scope of this paper. The fact that sometimes many properties are correlated makes the understanding of the physical mechanisms driving them difficult to discern. The Principal Component Analysis (PCA) is a statistical method which has proven to be very efficient at isolating the most important correlations in active galactic nuclei (AGN) (e.g. Boroson \& Green 1992). Here the PCA will be used with a BAL QSO sample.

Spectropolarimetry of BAL QSOs with a good signal to noise has been published for a large number of BAL QSOs (Ogle et al. 1999). In combination with our own spectropolarimetric data, we define spectropolarimetric indices to perform a statistical analysis. The results are then used in combination with previous analyses to better constrain the BALR models.

In Sect. 2, we describe the sets of data. The statistical analyses are done in Sect. 3. Section 4 is devoted to a principal component analysis of the data in order to identify the variables that correlate together and to relate them to more fundamental underlying parameters that drive the correlations. In Sect. 5, we present the new spectropolarimetry of 6 BAL QSOs. Considering additional data from the literature, spectropolarimetric indices are defined and correlations with broad band optical polarization and with optical indices are searched for. In the last section, we discuss and interpret the main results in the framework of existing BAL QSO models.

\section{The BAL QSO data}

The sample considered here is made up of 139 BAL QSOs. It is essentially a compilation of 93 BAL QSOs coming from large polarization surveys. In addition, we also consider 46 BAL QSOs with good quality optical (rest-frame UV) spectra but no polarization data. The optical polarimetric observations were conducted by us (Paper I; Lamy \& Hutsemékers 2000a; Sluse et al. 2004; Hutsemékers et al. 2004) and by Ogle et al. (1999) and Schmidt \& Hines (1999). The spectra considered for the measurement of optical indices are those from the W91 and Korista et al. (1993) samples (mainly drawn from the LBQS), and from the recent FIRST (Becker et al. 2000, 2001) and SDSS/FIRST (Menou et al. 2001) surveys. Apart from these major surveys, we do not consider individually studied BAL QSOs. Indeed such objects are not very numerous and are usually observed or detected because of their peculiar properties. For the same reason, we do not consider the unusual BAL QSOs uncovered by Hall et al. (2002).

The characteristics of the BAL QSOs considered in this study are reported in Table 1 and described in detail below.

\subsection{The broad band polarization data}

The broad band polarization data are compiled from our previous surveys (Paper I; Lamy \& Hutsemékers 2000a), from the survey of Schmidt \& Hines (1999) and from the spectropolarimetric atlas of Ogle et al. (1999). When an object is observed several times, we choose the value of the polarization degree with the lowest uncertainty, i.e. the smallest $\sigma_{\mathrm{p}}$. Our surveys have been conducted in the $\mathrm{V}$ filter with a typical uncertainty $\simeq 0.2 \%$. These uncertainties are adequate for BAL QSO studies since the polarization of these objects peaks around $\sim 1 \%$ (Paper I; Lamy 2003). The measurements of Schmidt \& Hines (1999), mostly obtained in white light, have often larger uncertainties $\left(\sigma_{\mathrm{p}} \simeq 0.4 \%\right)$. The data from Ogle et al. (1999) are high-quality broad-band polarizations (4000-8600 $\mathrm{A}$ ) measured from their spectropolarimetric data, and obtained either with the Keck or the Palomar telescopes. The typical uncertainties range from $\sim 0.05 \%$ for the Keck data to $\sim 0.15 \%$ for the Palomar data. Within the uncertainties, no polarization variability is observed for BAL QSOs with multiple observations. Finally, a few additional polarization measurements of BAL QSOs from the LBQS and FIRST surveys (Becker et al. 2000; Menou et al. 2001) have recently been secured using the ESO $3.6 \mathrm{~m}$ telescope at La Silla (Sluse et al. 2004; Hutsemékers et al. 2004) and are added to the sample.

The observed degree of polarization $p$ and its uncertainty $\sigma_{\mathrm{p}}$ are given in Table 1 together with the debiased degree of polarization $p_{0}$ according to the Wardle \& Kronberg (1974) method. Since most BAL QSOs in Table 1 have a polarization degree of the order of $1 \%$, we adopt a quality requirement on $p_{0}$ for all the statistical tests performed throughout this paper and involving this quantity. Data with poor signal-to-noise ratio are excluded by considering only those objects with $p_{0} / \sigma_{\mathrm{p}}>2$ if $p_{0} \geq 0.80 \%$ or with $\sigma_{\mathrm{p}}<0.40 \%$ if $p_{0}<0.80 \%$.

\subsection{The BAL QSO sub-types}

We use the classification described in detail in Paper I. After a careful visual inspection of the optical spectra available in the literature, each BAL QSO has been given a number between 2 and 6, according to the detection and intensity of the low ionization troughs. HIBAL QSO (type 2) are those objects which show broad absorption lines from highly ionized species but do not have broad absorption lines from $\mathrm{Mg}$ II nor Al III. If $\mathrm{Al} \mathrm{III}$ is not observed in absorption and if the optical spectrum does not cover the Mg II spectral region, the BAL QSO is considered as an unclassified BAL QSO (type 6). All the objects with either $\mathrm{Mg}$ II or $\mathrm{Al} \mathrm{III} \mathrm{broad} \mathrm{absorption} \mathrm{lines} \mathrm{in} \mathrm{their} \mathrm{spectra} \mathrm{are}$ classified as LIBAL QSOs. According to the strength of the low ionization absorption lines, we define three sub-categories of LIBAL QSOs: S (strong, type 3), W (weak, type 4) and M (marginal, type 5). Note that the term "LoBAL" widely spread in the literature essentially corresponds to our type 3 .

\subsection{Indices derived from the optical spectra}

We give in Table 1 the following indices originally defined by W91 to characterize the absorption/emission features in BAL QSOs: (1) the balnicity index BI which is a modified velocity equivalent width of the CIV BAL; (2) the half-width at half-maximum $H W H M$ and the rest equivalent width $R E W$ of the C III] $\lambda 1909$ emission line and of the red part of the C IV emission line; (3) the rest equivalent widths of emission features related to Fe II and located near 2070 and $2400 \AA$; and (4) the detachment index DI which measures the onset 
velocity of the strongest ${ }^{2} \mathrm{C}$ IV BAL trough in units of the adjacent emission line half-width, that is the degree of detachment of the absorption line relative to the emission one.

Apart from the W91 sample, none of these indices are given in the literature except BI. Therefore, as already done in Paper I and in Hutsemékers \& Lamy (2000, hereafter Paper II), we have computed these indices whenever possible by using good quality spectra digitally scanned from the literature. We follow the prescriptions given in W91 and refer the reader to this paper for additional details. The new measurements make use of spectra published by W91, Korista et al. (1993), Ogle et al. (1999), Becker et al. (2000, 2001), Menou et al. (2001), and a few spectra from the LBQS (Foltz et al. 1989; Chaffee et al. 1991; Hewett et al. 1991). For one object (B2240-3702), the indices have been measured from our spectra (cf. Fig. 3). All these new measurements are reported in Table 1 together with values previously published in Papers I and II.

In Table 1, following W91, we consider as BAL QSOs those objects with a non-zero $\mathrm{BI}$ in either $\mathrm{C} \mathrm{IV}$ or $\mathrm{Mg}$ II (since usually BI (C IV) $\geq \mathrm{BI}$ (Mg II)). But for the consistency of the statistical analysis, only the BI measured from C IV are considered. The BI values in Table 1 are mainly found in the literature (W91; Korista et al. 1993; Brotherton et al. 1998, 2002; Ogle et al. 1999; Becker et al. 2000, 2001; Menou et al. 2001). For the objects considered at the same time in the W91 and the Korista samples, we adopt the average of their values. We have calculated BI only for those objects observed at the Palomar Observatory by Ogle et al. (1999).

In addition to these indices, we have also reported in Table 1 the maximum velocity in the CIV BAL trough, $v_{\max }$, which provides an estimate of the terminal velocity of the flow. For the BAL QSOs not included in the samples of Papers I and II, the values of $v_{\max }$ are either given by Becker et al. (2000) and Menou et al. (2001), or they are evaluated from the Korista et al. (1993), Ogle et al. (1999) and Becker et al. (2001) spectra, by measuring, from the blue to the red, the wavelength at which the absorption first drops $10 \%$ below the flux level defined by the local continuum. At velocities higher than $\sim 25000 \mathrm{~km} \mathrm{~s}^{-1}$, C IV BALs may be contaminated by the Si IV emission line, such that measurements of $v_{\max }$ become inaccurate. In a conservative way, we therefore limit $v_{\max }$ to $25000 \mathrm{~km} \mathrm{~s}^{-1}$ from the CIV emission centroïd, even if larger values are sometimes given by some authors (Becker et al. 2000; Menou et al. 2001). In these cases, $25000 \mathrm{~km} \mathrm{~s}^{-1}$ constitutes a lower limit to the true $v_{\max }$. Finally, we have measured the slope of the continuum, $\alpha_{B}$, by fitting the continuum blueward of $\mathrm{C}$ III] and assuming a power-law continuum $F_{v} \propto v^{-\alpha_{B}}$ (see Paper I for details).

The errors associated with the measurements of these indices are difficult to estimate. They are essentially related to the positioning of the continuum but they also depend on the structure of the lines, the nominal redshift, etc. Although the uncertainty on the continuum is partly taken into account in its definition, errors on BI may still be large. We estimate them from values published by different authors (for example the

\footnotetext{
${ }^{2}$ In objects with multiple absorption troughs, W91 define the strongest absorption as the one with the largest equivalent width.
}

samples of W91; and Korista et al. 1993): typical uncertainties on BI are around a few hundred $\mathrm{km} \mathrm{s}^{-1}$. The typical errors on $v_{\max }$ have been estimated from two independent series of measurements and are typically around $1000 \mathrm{~km} \mathrm{~s}^{-1}$. The typical error on $\alpha_{B}$ is $\sim 0.3$, as evaluated by varying the possible continua (Paper I). The error associated with the detachment index DI is typically around $15 \%$ from a comparison between our values and those from W91, although it could be higher in some cases, especially for those objects with multiple troughs or complex absorptions. Indeed, depending on the continuum, one might select different troughs as being the strongest one. By doing the same kind of comparison with W91, we estimate the errors associated with CIV HWHM and CIV HREW to be $\sim 10 \%$ and $\sim 20 \%$ respectively.

Finally, we have included in Table 1 the $B$ absolute magnitude of the objects, $M_{B}$, which is calculated by following the prescriptions of Veron \& Veron (2000) (with $H_{0}=$ $50 \mathrm{~km} \mathrm{~s}^{-1} \mathrm{Mpc}^{-1}, q_{0}=0$ and an optical spectral index $\alpha=$ 0.3 ) and by applying the "BAL K-correction" of Stocke et al. (1992).

\section{Analysis of correlations involving the polarization and spectral indices}

Possible correlations involving the optical polarization and the indices defined in Sect. 2.3 have been searched for by computing the Kendall $\tau$ and the Spearman $r_{\mathrm{s}}$ rank correlation coefficients (Press et al. 1989). While our main goal is to investigate the behavior of the polarization, correlations between the indices themselves have also been searched for since several new measurements are available.

The results of these correlation tests are given in Table 2. The first two columns list the two indices we test. The next two columns give the probabilities $P_{\tau}$ and $P_{r_{\mathrm{s}}}$ to find a correlation by chance between two uncorrelated quantities. The column labeled $n$ is the number of objects involved in the analysis. Since we search for correlations among 11 quantities, a total of 55 correlations tests are performed. Since the number of objects is rather large for most pairs of indices, we may reasonably consider as significant only those correlations with $P \leq 0.02$. With 55 correlations tested, we expect to detect one false correlation with such a probability, assuming they are independent. In general, an excellent agreement is found between the results of the two statistical tests. The correlations have also been investigated for the HIBAL and LIBAL QSO subsamples separately and the results are given in Table 2 . A few correlations are detected only when considering the whole BAL QSO sample, illustrating the importance of the size of the sample.

Among all significant correlations reported in Table 2, several link in an obvious manner quantities either related by their definition or by a trivial common physical origin. In the first category, as an example, the correlation between BI and $v_{\max }$ simply reflects the fact that the wider the trough the larger both $\mathrm{BI}$ and $v_{\max }$ (with $\mathrm{BI} \leq v_{\max }-5000$ according to their definitions). Another such trivial correlation is DI - $v_{\max }$ : the most detached absorptions have necessarily large $v_{\max }$. In the second category, we may quote the correlations between C IV HWHM and C III] $H W H M$ and between the Fe II indices. Finally, a few 
Table 2. Analysis of correlations between $p_{0}$ and the indices described in Sect. 2.3 as well as correlations between the indices themselves. Three samples of BAL QSOs are considered. $n$ is the number of objects considered in the tests. The last column indicates whether the correlation is positive or negative (identical for the three BAL QSO samples). Boldfaced entries are the least likely to be due to random chance.

\begin{tabular}{|c|c|c|c|c|c|c|c|c|c|c|c|}
\hline \multirow[b]{2}{*}{ Index 1} & \multirow[b]{2}{*}{ Index2 } & \multicolumn{3}{|c|}{ BAL QSOs } & \multicolumn{3}{|c|}{ 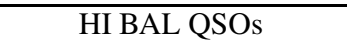 } & \multicolumn{3}{|c|}{ LI BAL QSOs } & \\
\hline & & $P_{\tau}$ & $P_{r_{\mathrm{s}}}$ & $n$ & $P_{\tau}$ & $P_{r_{\mathrm{s}}}$ & $n$ & $P_{\tau}$ & $P_{r_{\mathrm{s}}}$ & $n$ & \\
\hline$\overline{p_{0}}$ & $\mathrm{BI}$ & 0.176 & 0.180 & 58 & 0.557 & 0.646 & 27 & 0.153 & 0.180 & 21 & + \\
\hline$p_{0}$ & DI & 0.001 & 0.003 & 54 & 0.437 & 0.469 & 25 & 0.004 & 0.003 & 20 & - \\
\hline$p_{0}$ & C IV ншнм & 0.834 & 0.824 & 54 & 0.772 & 0.890 & 26 & 0.888 & 0.898 & 19 & - \\
\hline$p_{0}$ & C IV HREW & 0.113 & 0.099 & 53 & 0.538 & 0.560 & 25 & 0.078 & 0.046 & 19 & + \\
\hline$p_{0}$ & C III] ншнм & 0.059 & 0.085 & 48 & 0.744 & 0.859 & 24 & 0.322 & 0.458 & 16 & - \\
\hline$p_{0}$ & C III] REW & 0.305 & 0.305 & 33 & 0.763 & 0.770 & 15 & 0.170 & 0.308 & 12 & + \\
\hline$p_{0}$ & Fe II $\lambda 2400$ & 0.411 & 0.397 & 31 & 0.227 & 0.217 & 17 & 0.663 & 0.792 & 13 & + \\
\hline$p_{0}$ & Fe II $\lambda 2070$ & 0.022 & 0.033 & 38 & 0.007 & 0.003 & 17 & 0.516 & 0.541 & 15 & + \\
\hline$p_{0}$ & $\alpha_{B}$ & 0.082 & 0.081 & 49 & 0.030 & 0.024 & 22 & 0.517 & 0.494 & 18 & + \\
\hline$p_{0}$ & $v_{\max }$ & 0.978 & 0.967 & 57 & 0.656 & 0.631 & 27 & 0.710 & 0.627 & 21 & + \\
\hline BI & DI & 0.208 & 0.213 & 93 & 0.022 & 0.036 & 43 & 0.390 & 0.285 & 26 & + \\
\hline $\mathrm{BI}$ & C IV ншнм & 0.741 & 0.780 & 92 & 0.469 & 0.451 & 43 & 0.513 & 0.583 & 25 & + \\
\hline BI & C IV HREW & 0.495 & 0.405 & 90 & 0.965 & 0.869 & 42 & 0.301 & 0.209 & 25 & - \\
\hline BI & C III] ншнм & 0.044 & 0.030 & 69 & 0.100 & 0.093 & 41 & 0.384 & 0.409 & 18 & + \\
\hline BI & C III] REW & 0.505 & 0.466 & 38 & 0.888 & 0.912 & 19 & 0.273 & 0.354 & 12 & + \\
\hline BI & Fe II $\lambda 2070$ & 0.000 & 0.000 & 43 & 0.103 & 0.062 & 21 & 0.067 & 0.087 & 15 & + \\
\hline BI & Fe II $\lambda 2400$ & 0.000 & 0.000 & 35 & 0.008 & 0.006 & 21 & 0.197 & 0.254 & 13 & + \\
\hline BI & $\alpha_{B}$ & 0.003 & 0.003 & 66 & 0.708 & 0.741 & 32 & 0.021 & 0.016 & 24 & + \\
\hline BI & $v_{\max }$ & 0.000 & 0.000 & 108 & 0.000 & 0.000 & 53 & 0.030 & 0.058 & 30 & + \\
\hline DI & C IV ншнм & 0.527 & 0.509 & 91 & 0.200 & 0.224 & 42 & 0.513 & 0.619 & 25 & - \\
\hline DI & C IV HREW & 0.002 & 0.001 & 90 & 0.004 & 0.001 & 41 & 0.006 & 0.004 & 25 & - \\
\hline DI & C III] ншнм & 0.247 & 0.217 & 65 & 0.053 & 0.047 & 38 & 0.970 & 0.798 & 18 & + \\
\hline DI & C III] REW & 0.040 & 0.054 & 38 & 0.575 & 0.459 & 19 & 0.217 & 0.138 & 12 & - \\
\hline DI & Fe II $\lambda 2070$ & 0.335 & 0.310 & 43 & 0.305 & 0.207 & 21 & 0.520 & 0.576 & 15 & + \\
\hline DI & Fe II $\lambda 2400$ & 0.881 & 0.092 & 35 & 0.398 & 0.336 & 21 & 0.243 & 0.343 & 13 & + \\
\hline DI & $\alpha_{B}$ & 0.532 & 0.469 & 61 & 0.836 & 0.868 & 29 & 0.843 & 0.922 & 22 & - \\
\hline DI & $v_{\max }$ & 0.000 & 0.000 & 93 & 0.000 & 0.000 & 43 & 0.040 & 0.038 & 26 & + \\
\hline C IV ншнм & C IV HREW & 0.387 & 0.415 & 90 & 0.616 & 0.678 & 42 & 0.082 & 0.131 & 25 & + \\
\hline C IV ншнм & C III] ншнм & 0.004 & 0.005 & 64 & 0.105 & 0.148 & 37 & 0.017 & 0.011 & 18 & + \\
\hline C IV ншнм & C III] REW & 0.546 & 0.589 & 37 & 0.909 & 0.925 & 18 & 1.000 & 0.931 & 12 & - \\
\hline C IV ншнм & Fe II $\lambda 2070$ & 0.204 & 0.205 & 41 & 0.192 & 0.229 & 20 & 0.702 & 0.887 & 14 & + \\
\hline C IV ншнм & Fe II $\lambda 2400$ & 0.630 & 0.635 & 33 & 0.059 & 0.051 & 20 & 0.131 & 0.199 & 12 & + \\
\hline C IV ншнм & $\alpha_{B}$ & 0.442 & 0.380 & 59 & 0.858 & 0.843 & 28 & 0.080 & 0.038 & 21 & + \\
\hline C IV ншнм & $v_{\max }$ & 0.014 & 0.015 & 92 & 0.066 & 0.094 & 43 & 0.228 & 0.221 & 25 & + \\
\hline C IV HREW & C III] ншнм & 0.003 & 0.011 & 63 & 0.006 & 0.012 & 36 & 0.340 & 0.373 & 18 & - \\
\hline C IV HREW & C III] REW & 0.000 & 0.000 & 37 & 0.027 & 0.029 & 18 & 0.095 & 0.042 & 12 & + \\
\hline C IV HREW & Fe II $\lambda 2070$ & 0.498 & 0.525 & 41 & 0.845 & 0.885 & 20 & 0.868 & 0.976 & 14 & - \\
\hline C IV HREW & Fe II $\lambda 2400$ & 0.938 & 0.985 & 33 & 0.896 & 0.830 & 20 & 0.403 & 0.555 & 12 & - \\
\hline C IV HREW & $\alpha_{B}$ & 0.597 & 0.639 & 58 & 0.983 & 0.882 & 27 & 0.378 & 0.339 & 21 & - \\
\hline C IV HREW & $v_{\max }$ & 0.069 & 0.042 & 90 & 0.029 & 0.028 & 42 & 0.905 & 0.830 & 25 & - \\
\hline C III] ншнм & C III] REW & 0.512 & 0.498 & 38 & 0.916 & 0.932 & 19 & 0.272 & 0.183 & 12 & + \\
\hline C III] ншнм & Fe II $\lambda 2070$ & 0.275 & 0.228 & 41 & 0.952 & 0.867 & 21 & 0.088 & 0.061 & 13 & + \\
\hline C III] ншнм & Fe II $\lambda 2400$ & 0.852 & 0.867 & 33 & 0.504 & 0.482 & 21 & 0.392 & 0.247 & 11 & + \\
\hline C III] ншнм & $\alpha_{B}$ & 0.039 & 0.046 & 56 & 0.282 & 0.312 & 30 & 0.084 & 0.171 & 17 & + \\
\hline C III] ншнм & $v_{\max }$ & 0.050 & 0.063 & 69 & 0.156 & 0.237 & 41 & 0.099 & 0.092 & 18 & + \\
\hline C III] REW & Fe II $\lambda 2070$ & 0.920 & 0.912 & 38 & 0.574 & 0.557 & 19 & 0.411 & 0.457 & 12 & - \\
\hline C III] REW & Fe II $\lambda 2400$ & 0.943 & 0.997 & 30 & 0.207 & 0.193 & 19 & 0.025 & 0.038 & 10 & - \\
\hline C III] REW & $\alpha_{B}$ & 0.880 & 0.967 & 38 & 0.674 & 0.881 & 19 & 0.681 & 0.527 & 12 & + \\
\hline C III] REW & $v_{\max }$ & 0.125 & 0.117 & 38 & 0.379 & 0.501 & 19 & 0.726 & 0.678 & 12 & - \\
\hline Fe II $\lambda 2070$ & Fe II $\lambda 2400$ & 0.000 & 0.000 & 35 & 0.040 & 0.026 & 21 & 0.057 & 0.079 & 13 & + \\
\hline Fe II $\lambda 2070$ & $\alpha_{B}$ & 0.133 & 0.110 & 43 & 0.763 & 0.771 & 21 & 0.347 & 0.226 & 15 & + \\
\hline Fe II $\lambda 2070$ & $v_{\max }$ & 0.005 & 0.003 & 43 & 0.414 & 0.383 & 21 & 0.096 & 0.080 & 15 & + \\
\hline Fe II $\lambda 2400$ & $\alpha_{B}$ & 0.048 & 0.079 & 35 & 0.116 & 0.167 & 21 & 0.581 & 0.648 & 13 & + \\
\hline Fe II $\lambda 2400$ & $v_{\max }$ & 0.001 & 0.000 & 35 & 0.057 & 0.038 & 21 & 0.570 & 0.406 & 13 & + \\
\hline$\alpha_{B}$ & $v_{\max }$ & 0.001 & 0.001 & 66 & 0.280 & 0.226 & 32 & 0.008 & 0.005 & 24 & + \\
\hline
\end{tabular}



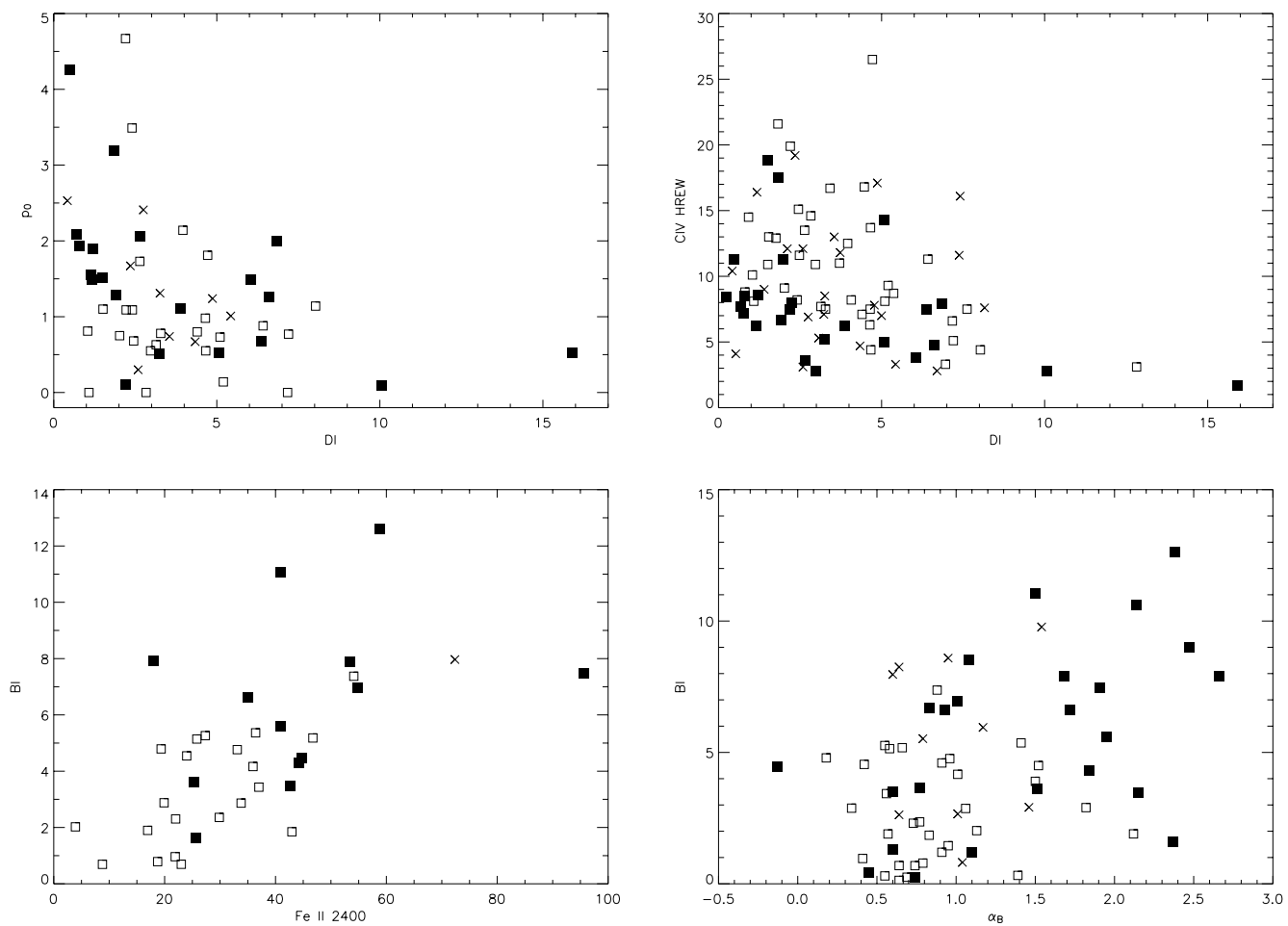

Fig. 1. The most significant non-trivial correlations (see text). $p_{0}$ is the debiased broad-band optical polarization (in \%), DI is the detachment index (unitless), C IV HREW is the rest equivalent width of the red part of the C IV BEL (in $\AA$ ), BI is the balnicity index (in $10^{3} \mathrm{~km} \mathrm{~s}^{-1}$ ), $\alpha_{B}$ is the slope of the continuum measured shortward of C III] (unitless) and Fe II 2400 is the rest equivalent width of the Fe II emission feature near 2400 Å. Open squares are HI BAL QSOs, filled squares are LI BAL QSOs and crosses are unclassified BAL QSOs.

correlations in Table 2 are due to the fact that two quantities are correlated with a third one: $v_{\max }$ is correlated with Fe II and $\alpha_{B}$ because, as we will see below, the balnicity index is correlated with these quantities. With all these arguments in mind, we are left with six significant non-trivial correlations: $p_{0}-\mathrm{DI}, \mathrm{BI}-$ Fe II $\lambda 2400, \mathrm{BI}-\alpha_{B}$, DI - C IV HREW, $p_{0}-$ Fe II $\lambda 2070$ and $\mathrm{C}$ IV $H W H M-v_{\max }$. They are all discussed in detail below and the most interesting ones are illustrated in Fig. 1. We have checked that these correlations remain significant when we exclude the objects with the most extreme properties (e.g. the two LIBAL QSOs with DI $\geq 10$ in Fig. 1a).

The degree of optical polarization, $p_{0}$, is essentially uncorrelated with most indices apart from a few exceptions. The most striking one is the anti-correlation between $p_{0}$ and the detachment index DI, already detected in Paper I and first suggested by Goodrich (1997). It is confirmed here with a higher significance $(P \simeq 0.002)$ and on the basis of a larger sample. It means that, in average, BAL QSOs with C IV P Cygni profile $^{3}$ are more polarized than objects with detached absorption troughs. As can be inferred from Table 2, this anti-correlation is strong for the whole BAL and the LIBAL QSO samples, but not detected in the HIBAL QSO sample. Figure 1 shows that the relation between $p_{0}$ and DI looks more like an inequality than a real anti-correlation. Indeed, for small values of DI, $p_{0}$ varies within a large range of values (from 0 to $5 \%$ ), while for large values of DI, $p_{0}$ is smaller. This correlation indicates

\footnotetext{
${ }^{3}$ DI is small in P Cygni type profiles since the absorption starts near zero velocity.
}

that DI may play a key-role in understanding the BAL QSO physics, as suspected by Hartig \& Baldwin (1986). Since it is the most important correlation involving polarization, it will be discussed in detail in Sect. 6.

The correlation between $p_{0}$ and Fe II $\lambda 2070$ is more doubtful because it is significant only for the HIBAL QSO subsample (marginal for the whole BAL QSO sample). Moreover, the fact that $p_{0}$ increases with the iron index does not support the usual interpretation of a dilution of $p_{0}$ by an unpolarized pseudo-continuum formed of a large number of metastable iron emission lines. Finally, we do not see any obvious reason why $p_{0}$ should correlate with Fe II $\lambda 2070$ and not with Fe II $\lambda 2400$. For all these reasons, we believe that this correlation is probably due to chance.

It is also important to remark that we do not detect any correlation between $p_{0}$ and BI. Such a correlation was reported by Schmidt \& Hines (1999) in their Fig. 8 (although their correlation was marginal with $P \sim 0.05$ ). Neither do we recover the correlation between $p_{0}$ and the slope of the continuum $\alpha_{B}$ discussed in Paper I and already interpreted as an artifact.

The correlations between BI and the Fe II $\lambda 2400$ index on one hand and between DI and C IV HREW on the other hand have been reported previously in W91. They are confirmed here with larger samples and with the use of BI averaged from the measurements of W91 and Korista et al. (1993). The correlation between BI and the Fe II $\lambda 2400$ index remains strong within the HIBAL QSO subsample while it disappears in the LIBAL QSOs subsample. These two correlations are illustrated 
in Fig. 1. Another significant correlation involving BI is the correlation between BI and the slope of the continuum $\alpha_{B}$ (see Fig. 1). It was found in Paper I and is confirmed here. It indicates that BAL QSOs with redder spectra display on average stronger absorption lines. Since these two correlations involving BI appear to be important in the description of the BAL phenomenon, they will be discussed later in the framework of the BALR models (Sect. 6.4).

The anti-correlation between DI and CIV HREW may possibly be explained in terms of radiative pumping of the BEL photons. Indeed, for $\mathrm{P}$ Cygni profiles $(\mathrm{DI} \ll)$, the BAL trough cuts off the blue wing of the C IV broad emission line (Turnshek 1988). Photons are absorbed and partly re-emitted in the red wing, therefore increasing the equivalent width of the red part of the C IV emission.

Finally, the correlation between C IV $H W H M$ and $v_{\max }-$ definitely less significant - is not obvious to interpret. Although it may be spurious, it may also suggest that the dynamics of the BAL wind is connected in some way to the dynamics of the broad emission line region from which it could be launched.

\section{Principal component analysis}

When we are dealing with a large number $n$ of BAL QSOs and a large number $p$ of distinct parameters characterizing the QSO properties, the study of the correlations two by two may be quite complex. Indeed, as can be seen from Table 2, several indices appear correlated, which makes it difficult to disentangle the leading correlations and consequently the physical mechanisms behind them. The Principal Component Analysis (PCA) is an alternative mathematical tool specifically designed to deal with such multivariate problems. Basically, the PCA identifies those parameters that correlate together and decreases the complexity of the problem by gathering them into only a few new parameters. In a $p$-dimensional space, PCA identifies those directions in which the cloud of points representing the QSOs is most elongated and uses them to define a new system of coordinates. Each axis in this new system is called an eigenvector and is simply a linear combination of the initial parameters. The first eigenvectors then contains most of the variance observed in the sample and identify the most significant correlations. For a complete mathematical description of multivariate statistics, see Murtagh \& Heck (1987). For nice illustrations of the application of PCA to some AGN data samples, see Boroson \& Green (1992), Francis \& Wills (1999) and Boroson (2002).

Since some objects have different wavelength coverages and/or their spectra are of poor quality, there are obviously missing data for many QSOs of our sample. Because a full data set is needed to run the PCA, the number of useful objects will then depend on the indices taken into account. As a rule of thumb, the more numerous the indices, the smaller the sample. Moreover, we wish to avoid most of the obvious correlations between closely related variables which would be dominant in the first eigenvectors. After several trials, we made a compromise between the size of the sample and the number of the adopted parameters. Together with $p_{0}$, all the parameters
Table 3. Results of the Principal Component Analysis. The first three principal components out of a total of 8 are given in order of their contribution to the total variance. The first row gives the variances of the data along the direction of the corresponding principal component. The sum of all the variances is equal to 8 , the number of variables considered in the analysis. The variance of each principal component is given as "proportion" in the second row and as "the cumulative proportion" in the third one. The rest of the table gives the correlation coefficients between the original variables and the principal components. Significant correlations are boldfaced, i.e. those ones with coefficients whose absolute values are $>1 / \sqrt{8}=0.3536$.

\begin{tabular}{lrrr}
\hline \hline & PC1 & PC2 & PC3 \\
\hline Eigenvalue & 2.63 & 1.92 & 1.27 \\
Proportion & 32.9 & 24.1 & 15.9 \\
Cumulative & 32.9 & 57.0 & 72.9 \\
Variable & PC1 & PC2 & PC3 \\
\hline$p_{0}$ & -0.1771 & $\mathbf{0 . 4 7 9 5}$ & $-\mathbf{0 . 4 2 9 7}$ \\
BI & $-\mathbf{0 . 5 0 6 5}$ & 0.2048 & -0.1247 \\
DI & -0.2440 & $-\mathbf{0 . 5 5 0 7}$ & -0.1789 \\
$\alpha_{\text {B }}$ & $-\mathbf{0 . 3 8 6 7}$ & 0.0654 & 0.1423 \\
C IV HREW & -0.0014 & $\mathbf{0 . 5 6 6 0}$ & -0.1301 \\
C IV нwнм & -0.1509 & 0.2636 & $\mathbf{0 . 7 2 1 8}$ \\
Fe II 2400 & $-\mathbf{0 . 4 8 6 5}$ & -0.1403 & -0.3062 \\
$v_{\text {max }}$ & $-\mathbf{0 . 4 9 3 5}$ & -0.1053 & 0.3406 \\
\hline
\end{tabular}

describing the emission and the absorption properties of the C IV line profile (BI, DI, C IV HREW, C IV HWHM and $v_{\max }$ ) are considered. Indeed, C IV measurements are available from medium resolution spectra for a large number of QSOs of our sample (W91; Korista et al. 1993). We also consider one of the Fe II intensity indices: Fe II 22400 . The Fe II $\lambda 2400$ index is favored since it is much stronger than the $\lambda 2070$ index. Finally, we add the slope of the continuum, $\alpha_{B}$, another important quantity in the description of BAL QSO spectral properties. The indices related to $\mathrm{C} \mathrm{III]} \mathrm{are} \mathrm{not} \mathrm{considered} \mathrm{because} \mathrm{of} \mathrm{the} \mathrm{well-}$ known blend with $\mathrm{Al}$ III and a possible blend with Fe III in some LIBAL QSOs (Hartig \& Baldwin 1986). Several PCA tests were performed including the absolute $\mathrm{B}$ magnitude of the objects. Since this quantity does not correlate with any of the indices, it may be safely discarded from the analysis. However, we cannot definitely rule out any correlation with the intrinsic luminosity since some BAL QSOs may be significantly reddened due to extinction (Sprayberry \& Foltz 1992; Reichard et al. 2003). The final sample then consists in 30 BAL QSOs with good quality measurements for the 8 variables previously described.

The PCA was carried out with the Fortran code written by Murtagh \& Heck (1987). Since the ranges of the variables are very different, the original data are first centred and reduced to unit standard deviation (correlation matrix option).

We present the results in Table 3 which lists the three most significant principal components (PC) and their projections upon the eight variables described above. Only the PCs with a variance (eigenvalue) larger than 1 are considered. More than half the total variance of the sample (57\%) is accounted for by the first two PCs which are elongated with variances larger than 
or of the order of twice that of the original data. We concentrate therefore exclusively on these two principal components which we call PC1 and PC2.

Apart from the usual redundant correlations, PC1 is dominated by the correlation between the balnicity index $\mathrm{BI}$ and the strength of the Fe II emission (and marginally $\alpha_{B}$ ), while PC2 correlates essentially $p_{0}$, DI and C IV HREW. This analysis is in good agreement with the classical correlation tests performed in Sect. 3. Indeed, two of the four important correlations discussed in Sect. 3 ( $p_{0}-\mathrm{DI}$ and DI - C IV HREW) dominate the eigenvector PC2 while a third one (BI - Fe II $\lambda 2400)$ dominates PC1. Small differences with the previous analysis (i.e. the significance of some correlations) may be explained in terms of the smaller sample considered here. Therefore, the main result emerging from the PCA is that BI, Fe II $\lambda 2400$ and maybe $\alpha_{B}$ on one hand, and $p_{0}$, DI and C IV HREW, on the other hand, vary together. As will be discussed in Sect. 6, PC1 could be connected to the accretion rate of matter (since this interpretation may qualitatively reproduce the correlation BI - Fe II), while PC2 could be related to the orientation of the BAL QSO with respect to the line of sight. The existence of two PCs outlines that, although important, orientation is probably not the only parameter governing the BAL QSO properties.

Figure 2 illustrates the distribution of the 30 BAL QSOs in the PC1-PC2 space. Different symbols have been used to separate the HIBAL and LIBAL QSO subsamples and to distinguish the lower luminosity objects (with $M_{B}>-28$ ) from the brighter ones (with $M_{B} \leq-28$ ). The LIBAL QSO subsample is essentially located in the $\mathrm{PC} 1 \leq 0$ region while most of the HIBAL QSOs have PC1 > 0. The HIBAL and LIBAL QSO subsamples are therefore better discriminated with PC1 than with PC2. Indeed, the LIBAL QSOs have the largest BI and Fe II emission or at least they display the most extreme values (see Fig. 1). If PC2 is indeed related to orientation, it plays a less important role in explaining the differences observed between HIBAL and LIBAL QSOs. Among the LIBAL QSOs, there is also a trend (not represented in Fig. 2) that the objects with stronger low ionization troughs have more negative values of PC1, i.e. the S LIBAL QSOs have more negative PC1 than $\mathrm{W}$ or M LIBAL QSOs, the latter having values of PC1 closer to those of HIBAL QSOs. Finally, interestingly enough, fainter QSOs appear to be located in the $\mathrm{PC} 1 \leq 0$ part of the diagram.

\section{BAL QSOs spectropolarimetry}

\subsection{Description of the observed sample}

Seven BAL QSOs were chosen for spectropolarimetry because of their brightness, their high degree of linear polarization in the $V$ filter (Paper I) and their redshift adequate for the observation of the C IV absorption line. Moreover, all these objects are LIBAL QSOs with strong, weak or marginal absorptions from low ionization species. They also span a range of other properties: B2225-0534 and B2240-3702 are BAL QSOs with $\mathrm{P}$ Cygni $\mathrm{C} I V$ profiles $(\mathrm{DI} \ll)$ while B1246-0542 and B1011+0906 are objects with detached C IV troughs $(\mathrm{DI} \gg)$. B1413+1143 is a gravitationally lensed QSO with evidence for a micro-lensing effect (Angonin et al. 1990;

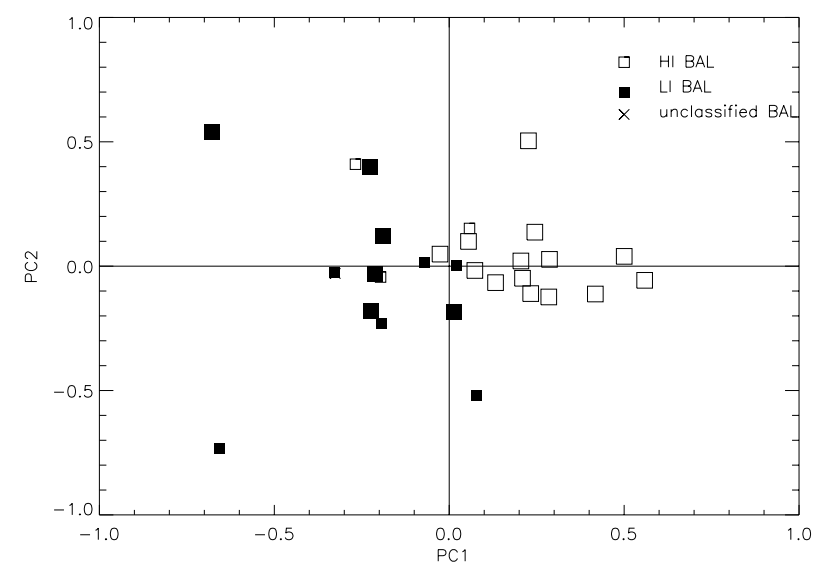

Fig. 2. Distribution of the $30 \mathrm{BAL}$ QSOs with respect to PC1 and PC2. Open squares represent HI BAL QSOs, filled squares LI BAL QSOs while the cross represents the only unclassified BAL QSO considered in the PCA. Large symbols refer to bright QSOs with $M_{B} \leq-28$ and small symbols represent fainter QSOs with $M_{B}>-28$.

Hutsemékers 1993) and B0059-2735 is the prototype of the so-called "Iron Lo BAL QSOs" (Hazard et al. 1987; Becker et al. 1997). Spectropolarimetry of B0059-2735 has been published elsewhere (Lamy \& Hutsemékers 2000b) and will not be discussed again.

Spectropolarimetry of B2225-0534 has been abundantly described in the literature in the last two decades (Stockman et al. 1981; Cohen et al. 1995; Goodrich \& Miller 1995; Ogle et al. 1999). Since there is no indication of variability in this object, these data have enabled us to check the whole observation/reduction procedure.

\subsection{Observations and data reduction}

The spectropolarimetric observations were carried out at the 3.6 m telescope (La Silla, ESO, Chile) equipped with EFOSC1 during two runs in April 5-8, 1995 and September 10-12, 1996.

Spectropolarimetry is performed with EFOSC1 by inserting a Wollaston prism in the filter wheel and a grism in the grism wheel (di Serego Alighieri 1998). The Wollaston prism splits the incoming radiation into two orthogonally polarized spectra separated by 20 .' Each object positioned on the slit has two spectra projected on the CCD detector. The measurement of their ratio at two different Wollaston angle positions separated by $45^{\circ}$ gives the two Stokes parameters describing the polarization (no half-wave plate was available at that time). In practice, the Wollaston prism does not move but rotates together with the whole EFOSC1 instrument, including the slit. In our case, this is not critical since our objects are point-like. In order to avoid overlapping of the object and sky spectra, a mask is placed in front of the slit; it is transparent only in slots whose length is equal to the beam separation produced by the Wollaston prism. The 2D image recorded on the CCD then consists of a number of alternate bands, two of them containing the two orthogonally polarized spectra of the object, the others being devoted to the sky. 
The detector was a $512 \times 512$ Tek CCD (ESO\#26) with an inverse gain of 3.8 electrons per ADU and a read-out-noise of 7.9 electrons. The pixel size was $27 \mu \mathrm{m}$ corresponding to 0.605 on the sky (Melnick et al. 1989). The slit had a width of 2 ". The seeing was typically around 1."4. The B300 grism was used for the seven objects giving a spectral coverage of 3600-6800 A. For two objects, the R300 grism was also used to enlarge the useful spectral range up to $\sim 8300 \AA$ allowing us to observe the Mg II BAL. The spectral resolution was $6.7 \AA$ per pixel. For each grism and position angle of the Wollaston, three frames were secured for a total exposure time of $90 \mathrm{~min}$ per Wollaston position. The splitting in shorter exposures ensures that the contamination by cosmic rays can be corrected.

Data reduction was accomplished with procedures developed within the ESO MIDAS package. The 2D raw data are first corrected for bias and flat-field. Cosmic ray events are interactively corrected and replaced by the median value of a small surrounding area. However, it is sometimes impossible to correct for some cosmic rays falling on the object spectra. These cosmic rays are easily detected by comparing the individual frames and they are flagged in the final 1D spectra with a "hit" label. The two object spectra and the four sky spectra (two on both sides of the object) are individually extracted with exactly the same procedure. The six spectra are calibrated in wavelength using $\mathrm{He}+\mathrm{Ar}$ internal lamp spectra. Since we observed small offsets between corresponding $\mathrm{He}+\mathrm{Ar}$ emission lines in orthogonally polarized spectra, six individual wavelength calibration curves were produced, and each object/sky spectra was independently wavelength calibrated. The sky spectra are averaged on both sides of the object and subtracted from the object spectra. Spectra are then corrected for the atmospheric extinction. Since there was no HWP plate in the setup, a flux calibration was done in order to correct for a possible difference of transmission of the Wollaston prism + grism for the ordinary and extraordinary rays. For that purpose, we observed spectrophotometric standard stars, assumed to be unpolarized, with the same instrumental setup. The standard star EG274 (Stone \& Baldwin 1983; Baldwin \& Stone 1984) was found to be very stable from night to night and was used to obtain the individual flux calibration curves. For that star, we measure a weak polarization degree $(p \leq 0.3 \%)$, more or less constant with wavelength, and in agreement with the observations by di Serego Alighieri (1994) with the same instrumental setup. Although the instrumental polarization induced by the instrument is therefore small, we took account of it by performing the flux calibration. Finally, it was necessary to rebin the data in wavelength in order to achieve a polarization uncertainty of $\sim 0.2-0.3 \%$ in the continuum. The spectra are rebinned on two original pixels, which corresponds to a spectral resolution of about $13 \AA$. The associated errors are calculated by propagating the errors from the photon noise in the object and sky spectra.

The Stokes parameters $q$ and $u$, the degree of polarization $p$ and the angle of polarization $\theta$ are computed from these rebinned spectra in the usual way (di Serego Alighieri 1998). Rebinning is done before the calculation of the Stokes parameters since $q$ and $u$ have non-gaussian distribution errors (Clarke $\&$ Stewart 1986). The photon noise is propagated throughout the equations in order to obtain $\sigma_{\mathrm{p}}$ and $\sigma_{\theta}$ in every bin of wavelength. Although $p$ is a well-known biased quantity (Simmons $\&$ Stewart 1985), we do not de-bias it since our data have large $\mathrm{S} / \mathrm{N}$ ratios. Finally, we calculate the polarized flux by multiplying the total flux by the degree of polarization. All these results are given in Figs. 3 and 4 for the six BAL QSOs.

A polarimetric standard star, HD 161291, was observed during both runs with the same setup. The interstellar polarization wavelength dependence was found in good agreement with that measured by di Serego Alighieri et al. (1994) using the same setup, and with the parameters given by Serkowski et al. (1975).

\subsection{Description of individual objects}

The spectropolarimetry of individual BAL QSOs is discussed in this section. We emphasize mainly their differences as well as new results that were not previously reported by Schmidt \& Hines (1999) and more particularly by Ogle et al. (1999) in their spectropolarimetric atlas. Note that Ogle et al. (1999) use $q^{\prime}$ instead of $p . q^{\prime}$ is the Stokes parameter $q$ rotated by the angle $2 \bar{\theta}$ where $\bar{\theta}$ is the mean continuum polarization angle. Although $q^{\prime}$ is unbiased, it has the disavantadge that its value is difficult to interpret when measured in emission or absorption lines with significant rotation of $\theta$.

1. B1246-0542 - This BAL QSO has deep broad absorption lines clearly detached from the corresponding emission lines. The polarization rises strongly in the C IV and Si IV broad absorption lines, mainly in the deepest troughs, while it slightly decreases in the emission lines. There is no rotation of the polarization accross those lines. The polarized flux of B1246-0542 is remarkable: first, it is nearly featureless with no trace of absorption, which is quite unusual among BAL QSOs (Schmidt \& Hines 1999; Ogle et al. 1999). Second, it shows an unusually large $\mathrm{N} \mathrm{V}$ emission, with no other emission lines. Apparently unnoticed, such a large $\mathrm{N} \mathrm{V}$ emission is in fact observed in the polarized flux of several BAL QSOs from the spectropolarimetric atlas of Ogle (1999), as well as in B1011+0906. A possible interpretation of this higher $\mathrm{N} \mathrm{V}$ emission in the polarized flux, in contrast with other lines such as $\mathrm{C}$ IV and Si IV, could be an extra N V emission due to resonance scattering of Ly $\alpha$ photons (Surdej \& Hutsemékers 1987), polarized due to a non-spherical geometry of the BALR (Lee \& Blandford 1997).

2. B1011+0906 - The spectrum of this BAL QSO has some similarities with B1246-0542, with deep absorptions well detached from relatively faint emission lines, and a strong $\mathrm{NV}$ emission. As usual, the polarization increases within the $\mathrm{C}$ IV trough. The polarized flux is characterized by the presence of a large N V emission. However, compared to B1246-0542, the polarized spectrum is complex and difficult to interpret. In particular, the polarization does not increase in the Si IV trough while there are some absorption seen in the polarized flux close to the location of the Si IV emission line. This latter absorption possibly results from C IV absorption at velocities larger than $25000 \mathrm{~km} \mathrm{~s}^{-1}$ which could be superimposed on the Si IV emission line. The Al III BAL is polarized as the continuum. 

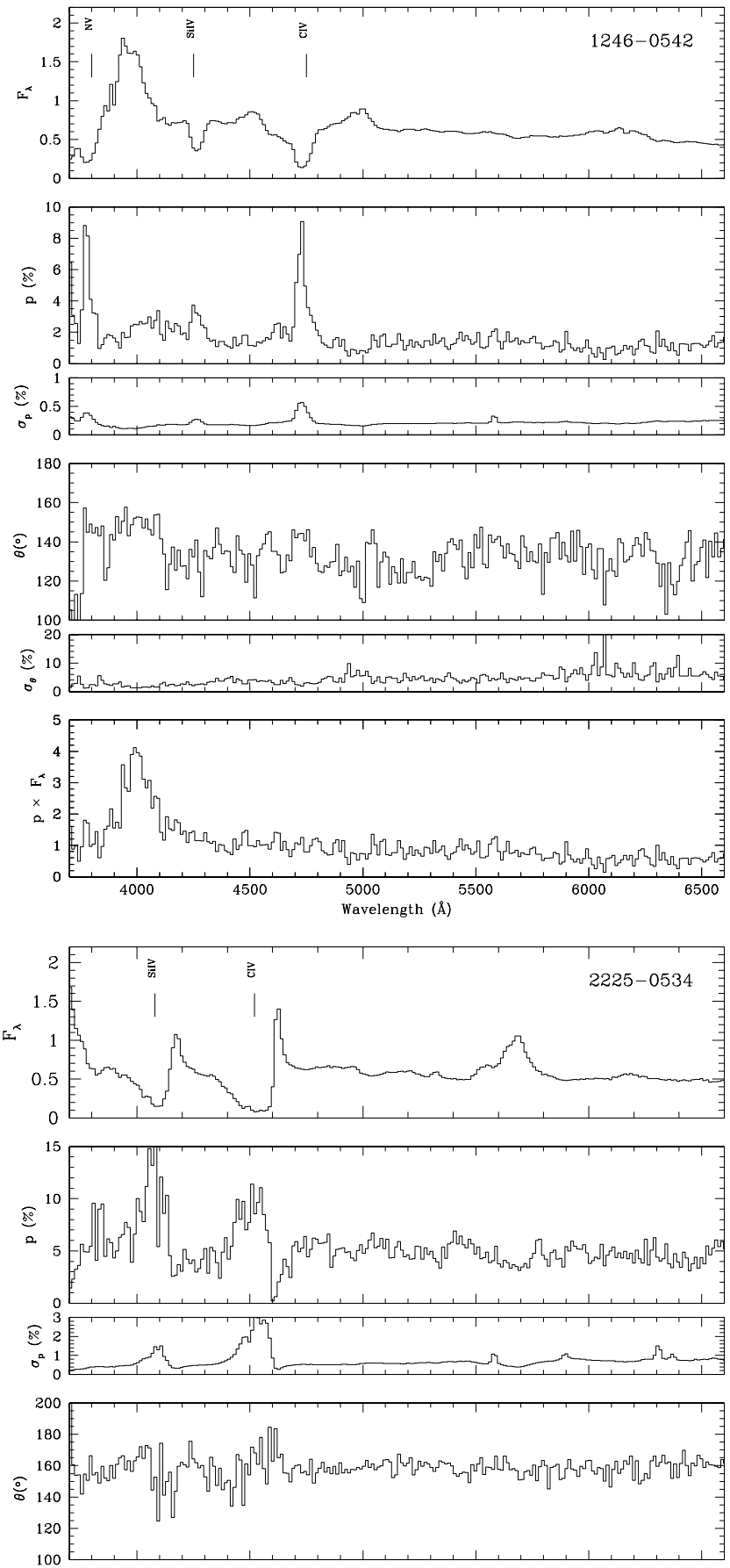

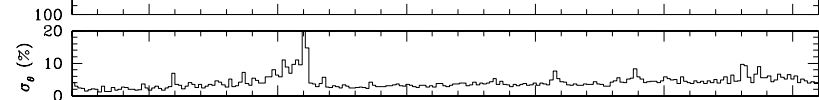

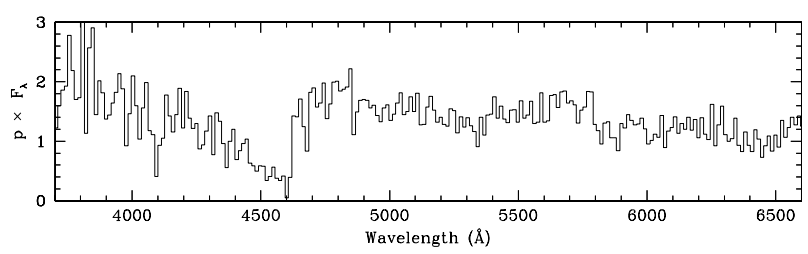

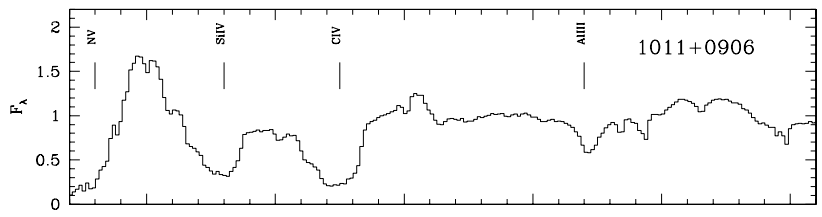
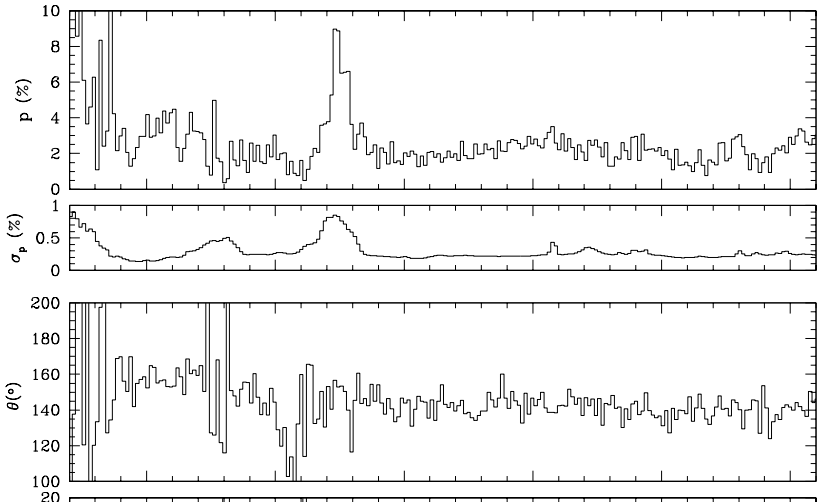

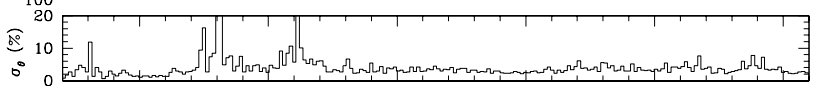
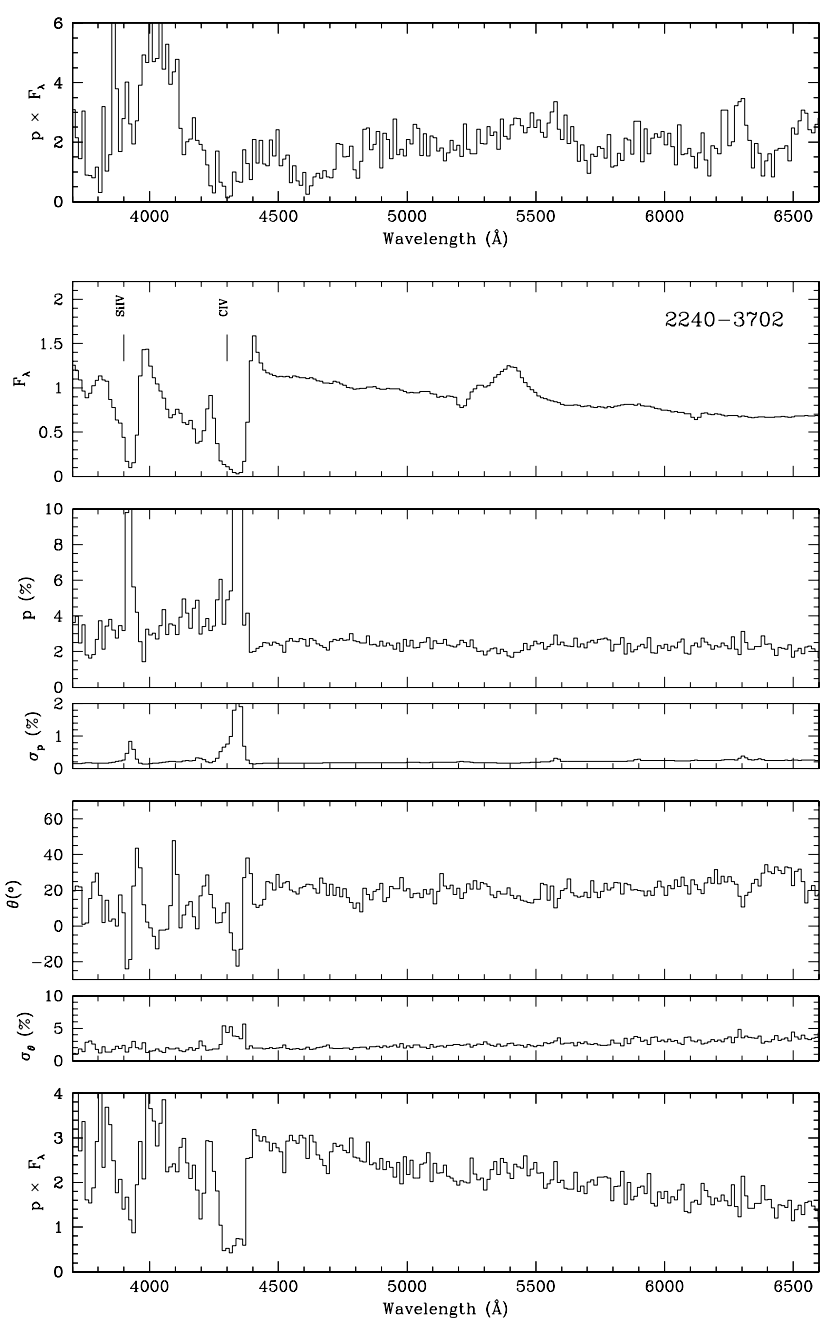

Fig. 3. Spectropolarimetric data for the BAL QSOs B1246-0542 (top left), B1011+0906 (top right), B2225-0534 (bottom left) and B2240-3702 (bottom right). For each object, there are six panels; from top to bottom: total flux $F_{\lambda}$, degree of polarization $p$, associated error $\sigma_{\mathrm{p}}$, polarization position angle $\theta$, associated error $\sigma_{\theta}$, and polarized flux $p \times F_{\lambda}$. $p$ has not been debiased. The main absorption lines are indicated. 

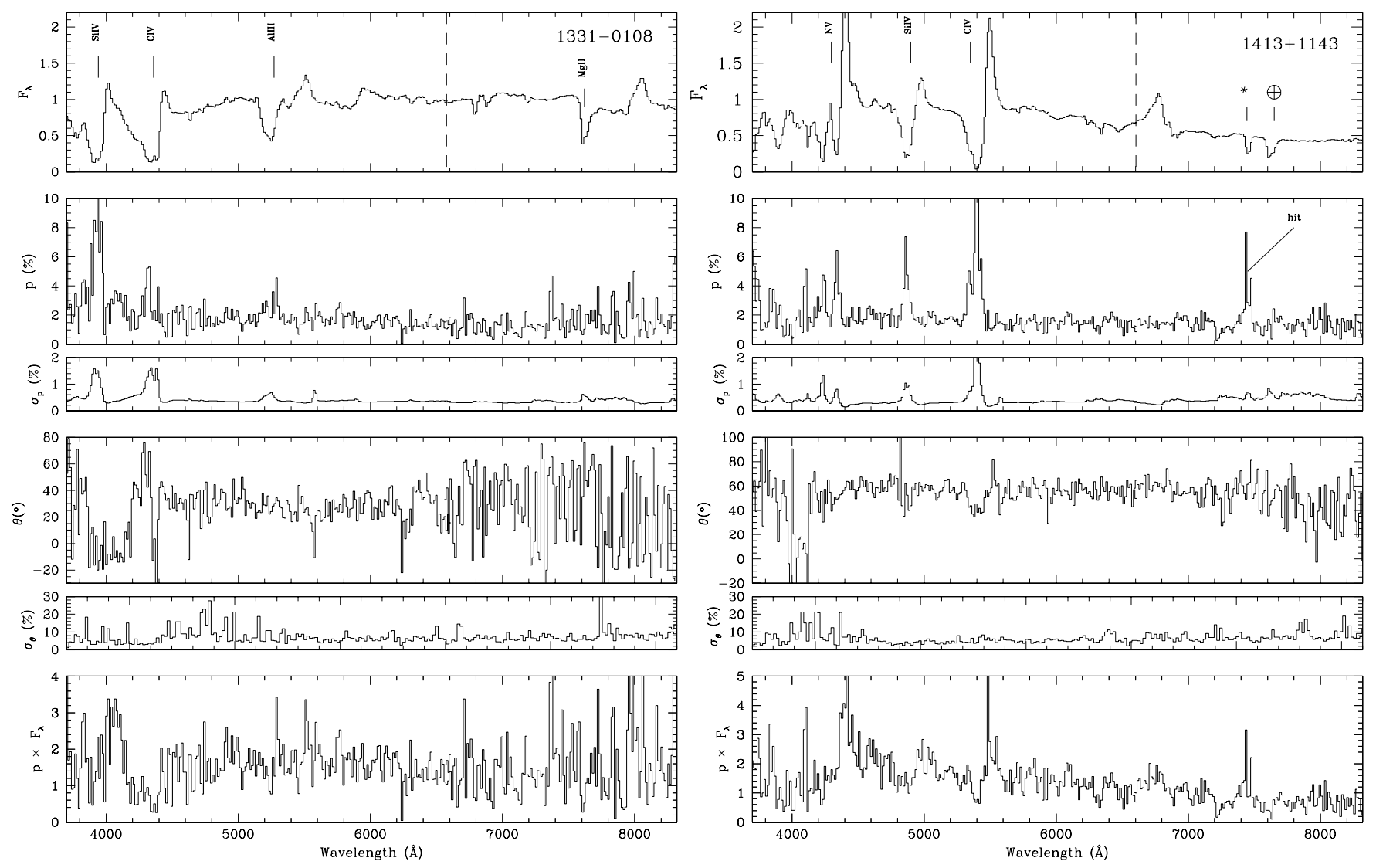

Fig. 4. Spectropolarimetric data for the BAL QSOs B1331-0108 (left) and B1413+1143 (right). Panels are as in Fig. 3. The B300 and R300 spectra were cut on their reddest and bluest parts respectively and merged together at the wavelength indicated by the vertical dashed line. The main absorption lines are indicated as well as features due to cosmic rays hits. The asterisk in the spectrum of B1413+1143 indicates an intervening absorption system at $z \sim 1.66$. The Earth symbol indicates a telluric absorption.

The rotation of $\theta$ near $4600 \AA$ is not significant at the $2 \sigma$ level, the uncertainty on $\theta$ in this region being large due to a decrease of $p$. However, if real, it may also be due to complex absorptions that are difficult to identify in this rather noisy part of the polarized flux.

3. B2225-0534 - This object is the prototype of the BAL QSOs with C IV P cygni type profiles. The spectropolarimetry of B2225-0534 has been published by Stockmann et al. (1981), Cohen et al. (1995), Goodrich \& Miller (1995) and Ogle et al. (1999). We confirm the main results found by these authors: the continuum is highly polarized with $p \sim 5 \%$, the broad emission lines are unpolarized with a large drop of $p$ in the C IV emission line, and the polarization strongly increases in the broad absorption lines. The polarization angle is constant throughout the spectral domain. The emission lines are absent from the polarized flux except a small residual from C III]. Since no indication of variability has been reported for this object by previous authors, the fact that our results are in very good agreement with theirs provides a useful cross-check of our reduction.

4. B2240-3702 - This BAL QSO is quite similar to B2225-0534. Indeed, it shows P Cygni type profiles and nearly black broad absorption lines. But B2240-3702 is also different with very weak emission lines and a double trough structure in the C IV and Si IV absorptions. The polarization strongly increases in the low velocity part of the absorption reaching a maximum of $\sim 25 \%$ in C IV. There is a dip of the polarization at the position of the emission lines. The polarization redward of C IV is flat $(p \sim 2 \%)$ with a small decrease at the location of $\mathrm{C}$ III]. The polarization angle rotates accross the broad absorption lines and is constant elsewhere. The broad absorption lines appear in the polarized flux although shallower. There is no evidence for any emission line in the polarized flux.

5. B1331-0108 - The data for this BAL QSO have a lower signal to noise ratio. There are however indications that the polarization increases in the C IV, Si IV and Al III absorption lines while there is no clear evidence for a rise of $p$ in the $\mathrm{Mg}$ II trough. Nothing can be said about the emission lines.

6. B1413+1143 ${ }^{4}$ - This BAL QSO is a four component gravitational mirage (Magain et al. 1988). Again, the polarization strongly increases in the absorption troughs. Although the absorptions of C IV and Si IV are nearly black in the total flux, they are shallower in the polarized flux. There is also a rotation of the polarization angle accross the C IV absorption line. Nevertheless, the most outstanding result in this object is the prominence of the emission lines in the polarized flux, indicating that they are polarized at a similar level as the continuum.

\footnotetext{
${ }^{4}$ Note that there is an error in the polarization position angle data of B1413+1143 displayed in Lamy \& Hutsemékers (1999).
} 
Table 4. The spectropolarimetric indices. The measurements were done using the spectropolarimetric data illustrated in Figs. 3 and 4 and the polarization spectra of Ogle et al. (1999). The continuum polarization, $p_{0}$, and its associated error, $\sigma_{\mathrm{p}}$, are taken from Table 1. $p_{0}$, $\sigma_{\mathrm{p}}$ and $p_{\max }$ are in $\%$ while the other quantities are unitless.

\begin{tabular}{lcrrrrr}
\hline \hline Object & $p_{0}$ & $\sigma_{\mathrm{p}}$ & $\begin{array}{r}p_{\mathrm{e}} / p_{\mathrm{c}} \\
\text { C IV }\end{array}$ & $\begin{array}{r}p_{\mathrm{e}} / p_{\mathrm{c}} \\
\mathrm{C} \text { III }\end{array}$ & SI & $p_{\max }$ \\
\hline B0019+0107 & 0.98 & 0.02 & 0.90 & 1.00 & - & $4.8 \pm 0.6$ \\
$\mathrm{~B} 0043+0048$ & 0.09 & 0.06 & - & - & - & $4.4 \pm 0.9$ \\
$\mathrm{~B} 0059-2735$ & 1.49 & 0.02 & - & - & - & $26.1 \pm 4.0$ \\
$\mathrm{~B} 0105-2634$ & 2.41 & 0.08 & 0.50 & 0.60 & 0.56 & $10.5 \pm 1.6$ \\
$\mathrm{~B} 0137-0153$ & 1.09 & 0.05 & 0.50 & 0.50 & - & $6.5 \pm 2.1$ \\
$\mathrm{~B} 0146+0142$ & 1.24 & 0.02 & 0.40 & 0.70 & 0.60 & $4.3 \pm 0.7$ \\
$\mathrm{~B} 0226-1024$ & 1.81 & 0.01 & 0.60 & 0.70 & 0.50 & $7.3 \pm 0.8$ \\
$\mathrm{~B} 0842+3431$ & 0.51 & 0.01 & 0.80 & 1.00 & 0.60 & $2.9 \pm 0.4$ \\
$\mathrm{~B} 0903+1734$ & 0.67 & 0.02 & 0.30 & 0.70 & - & $5.2 \pm 1.3$ \\
$\mathrm{~B} 0932+5006$ & 1.11 & 0.02 & 0.70 & 0.80 & 0.85 & $2.7 \pm 0.7$ \\
$\mathrm{~B} 1011+0906$ & 2.00 & 0.08 & 0.78 & 0.66 & 0.74 & $9.0 \pm 0.8$ \\
$\mathrm{~B} 1212+1445$ & 1.49 & 0.03 & - & 0.60 & 1.00 & $4.3 \pm 1.0$ \\
$\mathrm{~B} 1232+1325$ & 3.19 & 0.04 & 0.50 & 0.79 & 0.88 & $12.2 \pm 2.6$ \\
$\mathrm{~B} 1235+0857$ & 2.53 & 0.07 & - & 0.70 & 1.00 & $3.3 \pm 0.8$ \\
$\mathrm{~B} 1246-0542$ & 1.26 & 0.01 & 0.63 & 0.77 & 0.13 & $7.7 \pm 0.6$ \\
$\mathrm{~B} 1331-0108$ & 1.55 & 0.14 & 0.70 & 0.30 & - & $7.2 \pm 1.4$ \\
$\mathrm{~B} 1333+2840$ & 4.67 & 0.02 & 0.20 & 0.50 & 0.76 & $8.5 \pm 0.9$ \\
$\mathrm{~B} 1413+1143$ & 1.52 & 0.04 & 0.90 & 1.00 & 0.66 & $13.2 \pm 2.2$ \\
$\mathrm{~B} 1524+5147$ & 3.49 & 0.01 & 0.50 & 0.70 & 0.60 & $6.0 \pm 0.2$ \\
$\mathrm{~B} 2225-0534$ & 4.26 & 0.02 & 0.21 & 0.70 & 0.93 & $10.7 \pm 2.9$ \\
$\mathrm{~B} 2240-3702$ & 2.09 & 0.19 & 0.78 & 0.74 & 0.85 & $25.5 \pm 2.1$ \\
\hline & & & & & &
\end{tabular}

Only the semi-forbidden C III] line is absent in the polarized flux. Our results are in good agreement with those published previously by Goodrich \& Miller (1995), Schmidt \& Hines (1999) and Ogle et al. (1999), despite of the fact that the total flux and the polarization of $\mathrm{B} 1413+1143$ are known to be variable (e.g. Goodrich \& Miller 1995; Østensen et al. 1997).

\subsection{Definition of spectropolarimetric indices}

In this section we define four indices in order to describe the polarization of the emission and absorption lines in BAL QSOs. The measurement of these indices has been carried out on our spectropolarimetric data and on the spectra from Ogle et al. (1999). They are given in Table 4. For the five BAL QSOs of our sample with good quality data and which were also observed by Ogle et al. (1999) (i.e. B0059-2735, B1011+0906, B1246-0542, B1413+1143 and B2225-0534), the measurements are done independently for both data sets. Since they are in good agreement within uncertainties, we only give average values in Table 4.

The first two quantities measure the polarization in the C IV and $\mathrm{C}$ III] emission lines relative to the polarization of the redward adjacent continuum. They are given as $p_{\mathrm{e}} / p_{\mathrm{c}} \mathrm{C}$ IV and $p_{\mathrm{e}} / p_{\mathrm{c}} \mathrm{C}$ III] $]$ in Table 4 . The other two indices are related to the polarization in the $\mathrm{CIV}$ absorption line. The first one is the maximal polarization in the C IV trough, $p_{\max }$. The second one, denoted SI, is the ratio of the C IV absorption depths (measured at the wavelength of the deepest C IV absorption) in the polarized flux $p \times F_{\lambda}$ and in the total flux $F_{\lambda}$ where the continuum has been normalized to unity $\left(F_{\mathrm{c}}\right.$ is the flux in the continuum and $p_{\mathrm{c}}$ is the polarization of the continuum) i.e.,

$\mathrm{SI}=\frac{1-\frac{p \times F_{\lambda}}{p_{\mathrm{c}} \times F_{\mathrm{c}}}}{1-\frac{F_{\lambda}}{F_{\mathrm{c}}}}$.

Of course, we have $0 \leq \mathrm{SI} \leq 1$. SI is a measure of the strength of the absorption in the polarized flux with respect to the absorption in the direct flux.

The values given in Table 4 are measured for 21 objects with good signal to noise data. The uncertainties on $p_{\max }$ are taken from Ogle et al. (1999) or they have been measured from our data (Figs. 3 and 4). The uncertainties on the other indices are more difficult to estimate, namely since they depend on the position of the continuum and the signal to noise in the polarized spectrum. Based on two independent sets of measurements, we estimate that the typical uncertainties of these quantities are $\sim 10-15 \%$.

\subsection{Correlation analysis}

Table 5 gives the results of the correlation analysis performed between the four spectropolarimetric indices described in the previous section, the broad band polarization $p_{0}$ and three important and well-measured spectroscopic indices (BI, DI and $\alpha_{B}$ ) given in Table 1. Correlations have again been searched for using the Kendall $\tau$ and Spearman $r_{\mathrm{s}}$ rank correlation coefficients. With only 21 objects, we relax our criterion and consider as significant correlations with $P \leq 0.05$. With 22 correlations (assumed to be independent), we expect that one correlation with $P \leq 0.05$ is due to chance.

Although the relation $p_{\max }-\mathrm{BI}$ (see Fig. 5) is the most significant correlation in Table 5, we must consider it with caution. Indeed, $p_{\max }$ being measured where the absorption is the deepest, it may depend on the spectral resolution, more particularly when the residual intensity in the trough is nearly zero, which usually corresponds to large BI. In this case, it is possible that the higher the resolution, the lower the flux in a single pixel and the larger the measured $p_{\max }$. However, a majority of BAL QSOs with large BI do not have completely black troughs, and the $p_{\max }-\mathrm{BI}$ correlation is most probably real. This correlation is discussed in more details in Sect. 6.2.

As found in previous spectropolarimetric studies (e.g. Ogle et al. 1999), the polarization in the emission lines is systematically lower than that the polarization in the continuum, which corresponds to $p_{\mathrm{e}} / p_{\mathrm{c}} \leq 1$ for both the C IV and C III] emission lines (see Table 4). In addition, we find in Table 5 another significant correlation which relates the polarization in the CIV and C III] emission lines. As illustrated in Fig. 6, the polarization in the $\mathrm{C} \mathrm{III]}$ emission line is not only correlated to but also systematically higher than the polarization in the C IV emission line. If the broad emission lines appear in the polarized flux, one possible explanation may be the effect of two different polarizing mechanisms, one of them either 
Table 5. Results of correlation tests between spectropolarimetric indices, $p_{0}$ and three indices derived from optical spectra. $P_{\tau}$ and $P_{r_{\mathrm{s}}}$ give the probabilities that the correlation between the two indices are due to chance. $n$ is the number of points considered for the correlation. A correlation is emphasized if $P \leq 0.05$. The last column gives the sign of the correlation.

\begin{tabular}{|c|c|c|c|c|c|}
\hline Index 1 & Index 2 & $P_{\tau}$ & $P_{r_{\mathrm{s}}}$ & $n$ & \\
\hline$p_{0}$ & $p_{\mathrm{e}} / p_{\mathrm{c}} \mathrm{C}$ IV & 0.067 & 0.091 & 17 & - \\
\hline$p_{0}$ & $\left.p_{\mathrm{e}} / p_{\mathrm{c}} \mathrm{C}_{\mathrm{III}}\right]$ & 0.147 & 0.167 & 19 & - \\
\hline$p_{0}$ & SI & 0.224 & 0.254 & 15 & + \\
\hline$p_{0}$ & $p_{\max }$ & 0.029 & 0.019 & 22 & + \\
\hline$p_{\mathrm{e}} / p_{\mathrm{c}} \mathrm{C}$ IV & $\left.p_{\mathrm{e}} / p_{\mathrm{c}} \mathrm{C}_{\mathrm{III}}\right]$ & 0.012 & 0.018 & 17 & + \\
\hline$p_{\mathrm{e}} / p_{\mathrm{c}} \mathrm{C}$ IV & SI & 0.440 & 0.647 & 13 & - \\
\hline$p_{\mathrm{e}} / p_{\mathrm{c}} \mathrm{C} \mathrm{IV}$ & $p_{\max }$ & 0.966 & 0.936 & 17 & + \\
\hline$p_{\mathrm{e}} / p_{\mathrm{c}}$ C IV & BI & 0.523 & 0.583 & 17 & - \\
\hline$p_{\mathrm{e}} / p_{\mathrm{c}} \mathrm{C}$ IV & DI & 0.579 & 0.640 & 17 & + \\
\hline$p_{\mathrm{e}} / p_{\mathrm{c}} \mathrm{C}$ IV & $\alpha_{B}$ & 0.797 & 0.763 & 17 & + \\
\hline$\left.p_{\mathrm{e}} / p_{\mathrm{c}} \mathrm{C}_{\mathrm{III}}\right]$ & SI & 0.553 & 0.714 & 15 & - \\
\hline$p_{\mathrm{e}} / p_{\mathrm{c}} \mathrm{C}$ III] & $p_{\max }$ & 0.823 & 0.777 & 19 & - \\
\hline$p_{\mathrm{e}} / p_{\mathrm{c}}$ C III] & BI & 0.970 & 0.951 & 19 & + \\
\hline$\left.p_{\mathrm{e}} / p_{\mathrm{c}} \mathrm{C}_{\mathrm{III}}\right]$ & DI & 0.852 & 0.913 & 19 & + \\
\hline$p_{\mathrm{e}} / p_{\mathrm{c}} \mathrm{C}$ III] & $\alpha_{B}$ & 0.823 & 0.775 & 19 & - \\
\hline SI & $p_{\max }$ & 0.959 & 0.947 & 15 & - \\
\hline SI & BI & 0.919 & 0.914 & 15 & + \\
\hline SI & DI & 0.033 & 0.073 & 15 & - \\
\hline SI & $\alpha_{B}$ & 0.203 & 0.221 & 15 & + \\
\hline$p_{\max }$ & BI & 0.008 & 0.004 & 22 & + \\
\hline$p_{\max }$ & DI & 0.080 & 0.093 & 22 & - \\
\hline$p_{\max }$ & $\alpha_{B}$ & 0.089 & 0.059 & 22 & + \\
\hline
\end{tabular}

depolarizing C IV or polarizing C III]. Lee (1994) has shown that resonance scattering is more efficient at producing polarization in semi-forbidden lines such as C III] than in most permitted lines such as CIV. On the other hand, if there is only one polarizing mechanism at work for both emission lines, the emitting regions may have different geometries and/or sizes with respect to the scattering region (e.g. Goodrich \& Miller 1995). Cassinelli et al. (1987) have calculated the polarization of a spherical emission region of radius $\mathrm{R}$ scattered by a region located at a distance $D>R$. They find that the polarization should be corrected by a factor $\sqrt{1-(R / D)^{2}}$ compared to the case of a point source. If the size of the $\mathrm{C}$ III] emitting region is smaller than the size of the C IV emitting region, the geometric dilution may induce a larger polarization in the $\mathrm{C}$ III] emission line than in the C IV emission line. Of course, one must keep in mind that neither the broad emission line region nor the scattering region are likely to be spherical. Since these results are in contradiction with reverberation mapping studies (Clavel et al. 1991; Chiang \& Murray 1996), this explanation based on a purely geometrical dilution seems unlikely. In some BAL QSOs, the broad emission lines do not appear in the polarized flux indicating that they are not polarized at all. In this case, the observed correlation may simply reflect the fact that the polarization in the continuum is larger in the blue part of

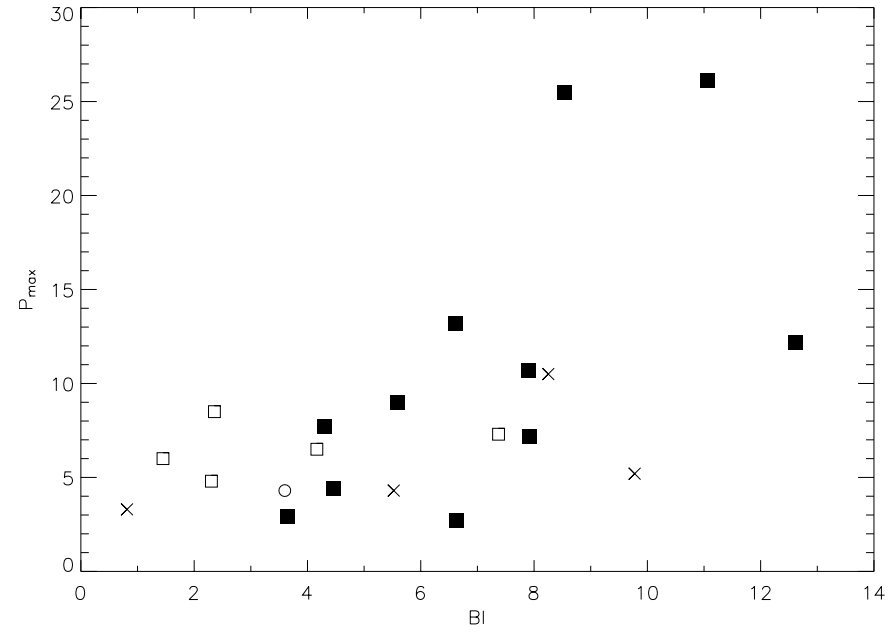

Fig. 5. The correlation between the maximum value of the polarization in the CIV absorption line and the balnicity index BI. Open squares are HIBAL QSOs, filled squares are LIBAL QSOs and crosses are unclassified BAL QSOs. The open circle corresponds to an uncertain measurement (see text).

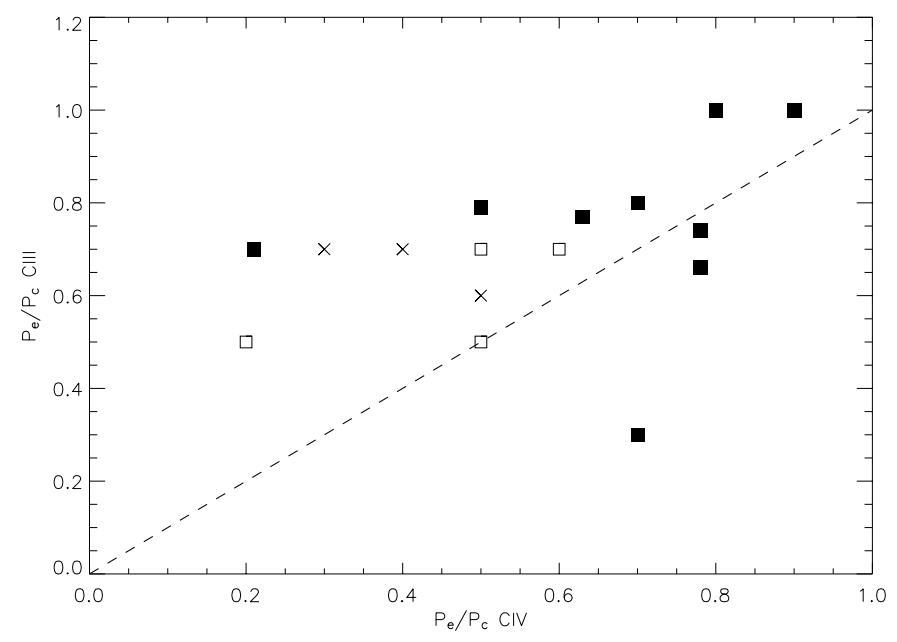

Fig. 6. The correlation between the polarization degrees in the C IV and $\mathrm{CIII}$ ] emission lines relative to the polarization in the adjacent redward continuum. The dashed line refers to an equal polarization in the two lines. Symbols are identical to those used in Fig. 5.

the spectrum (i.e. at the wavelength of CIV) than in the red part (i.e. at the wavelength of $\mathrm{C}$ III]).

There is also a marginal $(P \simeq 0.03)$ correlation between the detachment index and SI, which is worth considering. In Fig. 7, we plot SI versus DI. It clearly appears that if we do not consider the object represented by an open circle ${ }^{5}$, the correlation between SI and DI is much more significant. Indeed, in this case, the Kendall $\tau$ and the Spearman $r_{\mathrm{s}}$ statistics give a probability lower than 0.005 that these two quantities are correlated by chance. Additional measurements are badly needed to confirm this possible correlation. The correlation between SI and DI indicates that in P Cygni type BAL QSOs, the BALs

\footnotetext{
5 By carefully inspecting the data of Ogle et al. (1999), we noticed that the C IV absorption line in B1212+1445 is partly cut at the lower end of the spectrum such that the value of SI given in Table 5 for this object might be highly uncertain.
} 


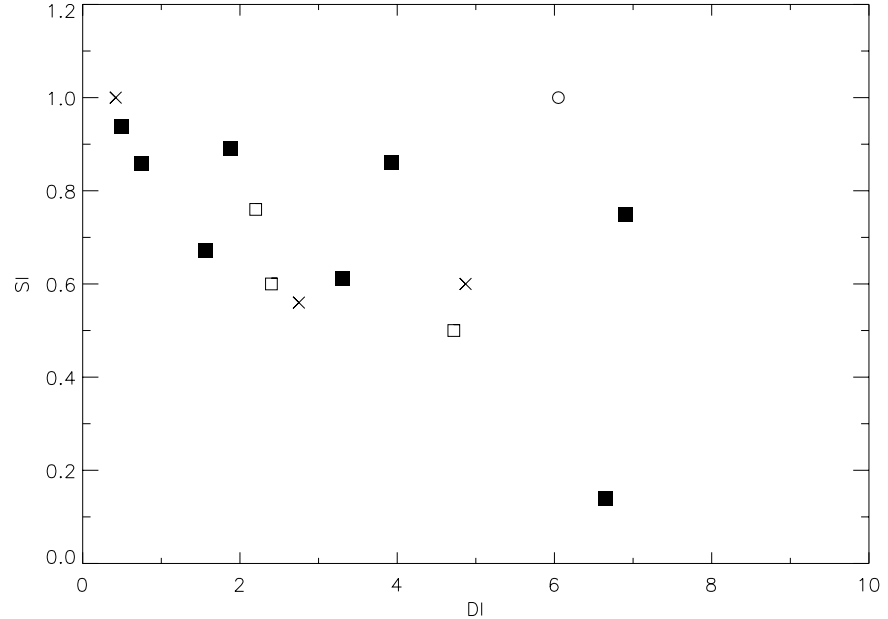

Fig. 7. The correlation between the detachment index DI and SI, the ratio of the C IV absorption depths in the polarized flux and in the total flux. Both quantities are unitless. Symbols are identical to those used in Fig. 5. The open circle corresponds to an uncertain measurement (see text).

in the polarized flux are nearly as deep as in the direct flux (cf. B2225-0543), while in BAL QSOs with detached troughs, the BALs in the polarized flux are much shallower than in the direct flux (cf. B1246-0542). As discussed in the next section, the consequences of such a relation for constraining BAL QSO models may be important.

\section{Discussion}

\subsection{The principal component PC2: The anti-correlation $p_{0}-D I$}

The fact that $p_{0}$ is only correlated with the detachment index emphasizes the importance of DI to understand the geometry of the outflow in BAL QSOs. The PCA has confirmed the importance of the $p_{0}-\mathrm{DI}$ anti-correlation which is dominant in PC2, one of the two significant principal components.

The WfD model of Murray et al. (1995) proposes a natural explanation of DI, hence of the detached troughs observed in some BAL QSOs (see Fig. 8). According to this model, an observer looking through the wind close to the disk (edge-on) sees absorption at all velocities including at low velocity. P Cygni type profiles are then produced and the observer measures a small value of DI. On the contrary, if the line-of-sight towards the observer grazes the upper edge of the wind, it misses the low velocity part of the absorption, the wind streamlines being more vertical than radial at low velocities. Therefore the absorption may start several thousand kilometers per second blueward of the corresponding emission peak, resulting in detached troughs and large values of DI. In the WfD model, DI is naturally related to the orientation of the accretion disk with respect to the line of sight. On the other hand, in the torus model (Schmidt \& Hines 1997), DI has no simple explanation. In polar wind models such as the one described by Punsly (1999), the detached troughs are caused by intermittent variations of matter ejections along the bipolar outflow. In this case,

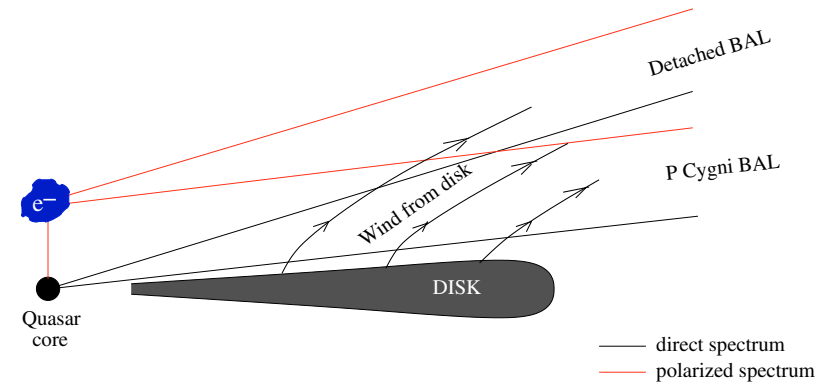

Fig. 8. Schematic illustration of the SI-DI relationship. The equatorial wind, represented by the curved streamlines, emerges from a geometrically thin accretion disk located in the equatorial plane. A mirror of electrons is located in the polar region and scatters some radiation coming from the central regions along the line-of-sight. This polarized component is then subsequently absorbed in the equatorial wind. When the disk is seen edge-on, P Cygni type profiles are observed since the observer sees absorption at all velocities including the low ones near the disk. For less inclined line-of-sights, detached absorption profiles are observed since the observer misses the low velocity part of the absorption.

the line detachment is related to a time-dependent phenomenon and does not depend on orientation.

Since the continuum polarization may also depend on the inclination, the relation between $p_{0}$ and DI favours a BALR model which is orientation dependent. In the WfD model, the dependence of $p_{0}$ with DI is naturally explained since higher polarization is expected close to the disk where attenuation of the direct unpolarized continuum is larger. As the inclination (measured from the disk axis) decreases, the growing importance of the direct continuum gives rise to a dilution of the polarization. Therefore, detached troughs are naturally associated with lower optical polarization. Since the polarization is not correlated with the slope of the continuum, it rules out an extinction by small dust particles as being the dominant extinction mechanism. A significant part of the attenuation should then be neutral, dominated either by electron scattering or by extinction by large dust particles.

The scatterers at the origin of the polarization may lie in the inner parts of the wind itself or they may be located in a polar region, which is usually assumed to interpret the spectropolarimetric observations (e.g. Hines \& Wills 1995; Goodrich \& Miller 1995), although its origin is unclear.

If the scatterers are located in the wind, the electron scattering model within the cylindrical sector geometry depicted by Brown \& Mc Lean (1977) may be considered to explain the $p_{0}-$ DI anti-correlation (see also Paper I). More particularly, as discussed in Sect. 3, Fig. 1a shows that the relation $p_{0}-\mathrm{DI}$ is not a true anti-correlation: for low values of DI (P Cygni profiles), there is a large range of $p_{0}$ values. At a given inclination, the Brown \& Mc Lean (1977) model predicts a polarization which increases with the half-opening angle of the wind. The range in $p_{0}$ for a given inclination may therefore simply reflect a range of opening angles of the equatorial wind. For large values of DI (profiles with detached troughs), the strong dilution by the direct unpolarized continuum leads to small values of $p_{0}$ whatever the opening angle of the wind. The larger polarization 
of LIBAL QSOs compared to HIBAL QSOs (Paper I; Lamy 2003) may then be explained either by the stronger extinctions observed in LIBAL QSOs (Sprayberry \& Foltz 1992; Reichard et al. 2003) or by a larger opening angle of their wind. This latter possibility is in agreement with the high covering factors needed to interpret their very weak [O III] emission (Boroson \& Meyers 1992; Yuan \& Wills 2003). This simple explanation qualitatively reproduces the observed $p_{0}-$ DI correlation. For HIBAL QSOs, the correlation is possibly weaker because of the variation from object to object such that the smaller range of $p_{0}$ values may mask the correlation.

\subsection{The anti-correlation $S I-D I$ and the correlation $p_{\max }-B I$}

If the scatterers are located in a polar region, part of the light from the continuum may be scattered and subsequently absorbed in the equatorial wind. The variation of SI with DI, derived from the spectropolarimetric data, is naturally explained within this geometry, as illustrated in Fig. 8: for nearly equatorial line-of-sights $(\mathrm{DI} \ll)$, the scattered flux crosses regions of the equatorial wind located close to the accretion disk where the opacity in the absorption lines is large. Therefore the polarized flux is significantly absorbed and shows deep broad absorption lines (SI 1, cf. B2225-0534). For less inclined line-of-sights (DI»), the scattered flux crosses regions of the equatorial wind where the opacity is much lower resulting in weaker absorptions in the polarized flux $(\mathrm{SI} \ll)$. The quantity SI becomes $\simeq 0$ in the extreme case where the scattered flux is not absorbed at all (cf. B1246-0542). It is more difficult to understand this relation if the scatterers are located in the equatorial wind itself than in a polar region. The SI-DI relationship appears therefore important for constraining the geometry of the BALR, and is definitely worth confirming with additional spectropolarimetric observations.

The other correlation derived from the spectropolarimetric data, $p_{\max }-\mathrm{BI}$, may also be simply explained with such a model. If $\mathrm{BI}$ is large, the direct unpolarized flux is strongly absorbed in the line resulting in a smaller dilution of the scattered component at these wavelengths. Therefore large values of $p_{\max }$ are expected in the case of strong absorptions.

\subsection{A "two-component wind" model?}

Although the $p_{0}-$ DI and SI - DI anti-correlations can be interpreted assuming an equatorial wind issued from a disk, the existence of an additional polar region where scattering takes place is apparently needed, at least in some BAL QSOs. This additional region may simply consist of another part of the outflow with a lower density and a larger amount of free electrons. Since the properties of these possibly related regions are different, we call hereafter this model the "two-component wind" $(2 \mathrm{CW})$ model, emphasizing that it is a natural extension or a variant of the WfD model.

The 2CW model is supported by several theoretical models which have been developed in the last years (Proga et al. 1998, 1999; Proga 2000, 2003; Pereyra et al. 2004). These authors generalize the Murray et al. model, in particular by including a magnetic field distribution. The outflow may then be radiation or magnetically driven or both. The simulations show that different types of winds can be produced (from being nearly polar to nearly equatorial) by varying the magnetic field, the total luminosity or the ratio between the luminosity of the disk and that of the central compact object. In particular, one may qualitatively reproduce a $2 \mathrm{CW}$ model with a dense equatorial wind bounded on the polar side by a much less dense stream.

Observationally, the rotation of the polarization angle $\theta$ accross broad absorption lines (a remarkable instance is given in B1413+1143, see Fig. 4) may provide evidence for the existence of two scattering regions in some BAL QSOs. Indeed, the continuum may be polarized by scattering from a polar and an equatorial distribution of electrons (or dust) at the same time. The scattered flux from the polar region has a polarization angle perpendicular to the disk axis while the polarization produced in the disk is parallel. The resulting polarization angle depends on the relative importance of both components. If the scattered flux from the polar region is re-absorbed in the equatorial wind, its contribution is reduced at these wavelengths leading to the observed rotation of the polarization angle. On the other hand, there may be only one source of scatterers, and the rotation of $\theta$ may result from another polarizing mechanism, for example resonance scattering since in P Cygni type profiles the broad absorption is partly filled with resonantly scattered emission.

Besides polarization, another indicator of geometry is the radio morphology. Some observational evidence supporting the existence of much smaller and weaker radio jets in radio-quiet QSOs have recently emerged (Kuncic 1999). Since the axis of the accretion disk is usually thought to be aligned with the radio jets, the comparison of the position angles of radio axes (PA) and optical polarizations $(\theta)$ can in principle give clues on the location of the scattering region. If the broad absorption lines occur in an equatorial wind, BAL QSOs are quasars seen edgeon and we should detect a radio axis close to the plane of the sky. The optical polarization should be roughly parallel to the radio axis if the scatterers are located in the wind or roughly perpendicular to it if they lie in a polar region. Unfortunately, because of their weak radio fluxes and their large distance, there are very few BAL QSOs with a resolved radio structure. In the low redshift BAL QSO PG 1700+5153, Kellermann et al. (1994) measure $\mathrm{PA} \sim 145^{\circ}$ for the radio structure observed at the VLA while Ogle et al. (1999) and Schmidt \& Hines (1999) measure a polarization position angle $\theta \sim 53^{\circ}$. In the radio-loud BAL QSO LBQS 1138-0126, Brotherton et al. (2002) observe a double-lobed radio structure with $\mathrm{PA} \sim 52^{\circ}$ characteristic of an edge-on source in unified radio schemes. Their spectropolarimetry of LBQS $1138-0126$ shows a continuous rotation of the continuum position angle from $180^{\circ}$ in the blue to $150^{\circ}$ in the red part of their optical spectra. In both objects, the optical polarization is then more or less perpendicular to the radio structure, suggesting that the scatterers are located in a polar region. However, we must keep in mind that at the VLA resolution, the weak radio axis may be twisted by some interactions with the surrounding medium. Therefore, higher resolution images are needed to confirm the VLA results. Recently, 
Jiang \& Wang (2003) performed the first VLBI observations of three BAL QSOs. Among them, J 1312+2319 has a two-sided structure with a position angle $\mathrm{PA} \sim 59^{\circ}$ indicating an edge-on orientation. We have recently obtained the optical polarization of this object for which we find $\theta \sim 166^{\circ}$ (Sluse et al. 2004) indicating that the two position angles are roughly perpendicular. Although there are only very few radio observations of BAL QSOs, they apparently support a $2 \mathrm{CW}$ model, where scattering occurs in a polar region.

In the $2 \mathrm{CW}$ model, the BALR is located in the equatorial plane. However, based on radio observations of FIRST BAL QSOs with the VLA, Becker et al. (2000) argue that BAL QSOs are not viewed from any particular orientation because they observed both steep and flat spectra within a sample of BAL QSOs discovered in the FIRST catalog. While, in BAL QSOs, it is not sure that the radio spectrum is a good indicator of the position angle of the radio axis as it is in radioloud sources, the recent VLBI observations of Jiang \& Wang (2003) may confirm the claim of Becker et al. (2000). Indeed, while J $1312+2319$ is seen edge-on, J 1556+3517 has a compact structure and a flat radio spectrum suggesting it is probably seen pole-on. However, J $1556+3517$ has also very unusual spectropolarimetric properties (Brotherton et al. 1997), possibly making it a peculiar object.

\subsection{The principal component PC1: The correlation $\mathrm{BI}-\mathrm{Fe}$ II}

Until now, we have concentrated on PC2 which is most probably driven by orientation. But what drives PC1 in BAL QSOs? Yuan \& Wills (2003) have shown that $z \sim 2$ BAL QSOs follow the main correlations linking the properties of emission lines as depicted in the so-called Boroson \& Green eigenvector (1992). In particular, BAL QSOs have the strongest Fe II and weakest [O III] emission among high redshift quasars. For nonBAL QSOs, these properties are usually interpreted in terms of accretion rates of gas around the central massive black hole (Boroson \& Green 1992). When the accretion rate increases, the disk inflates because of the larger radiation pressure. The optical depth becomes higher and the optically thick gas prevents the UV ionizing photons to reach the narrow-line region where [O III] forms. At the same time, the Fe II emission strongly increases because of a larger amount of X-rays. In this scenario, BAL QSOs are quasars which accrete matter with a rate close to the Eddington rate (Yuan \& Wills 2003) such that they display the most extreme Fe II and [O III] emission properties. In the WfD model, it is possible that the larger the accretion rate, the higher the gas density in the wind and therefore the stronger the absorption. This could possibly explain the positive correlation observed between BI and Fe II $\lambda 2400$, which dominates PC1.

\section{Conclusions}

With new broad-band polarization data and new measurements of indices on good quality spectra from the literature, we have performed a systematic search for correlations among a sample of 139 BAL QSOs. We find six non-trivial correlations.
We use a principal component analysis to isolate the main correlations in only two principal components: PC1, dominated by the $\mathrm{BI}-\mathrm{Fe}$ II correlation, is possibly related to the accretion rate of matter around the central massive black hole, while PC2, dominated by the $p_{0}-\mathrm{DI}$ anti-correlation, could be driven by orientation.

We have also searched for correlations among indices measured on spectropolarimetric data for a sample of 21 BAL QSOs. We find that the polarization in the C III] emission line is systematically larger than the polarization in the C IV emission line, and that the maximum of the polarization in the C IV absorption line is higher for BAL QSOs with large values of BI. Another important result is a possible correlation between the detachment index DI and SI, a quantity which measures the depth of the C IV BAL in the polarized flux relative to that one in the total flux. These statistical studies outline the importance of polarization to disentangle the geometry of the outflows in BAL QSOs. We have also emphasized that BI and more particularly DI are critical parameters to describe the BAL phenomenon.

The anti-correlations $p_{0}-$ DI and DI-SI are naturally explained with a model where the continuum attenuation and the line absorption occur within a continuous equatorial wind accelerated from a disk. The direct continuum is also assumed to be scattered by a "mirror" of electrons located in polar regions, possibly belonging to a polar wind. For edge-on line-of-sights, the direct unpolarized continuum is attenuated in the dense equatorial regions near the disk. Therefore, in BAL QSOs with P Cygni profiles, a large continuum polarization is observed because the relative contribution of the scattered polarized component is large. The scattered flux is also absorbed in the equatorial wind and a prominent absorption line is observed in the polarized spectrum. For a less inclined lineof-sight grazing the upper edge of the equatorial wind, the direct unpolarized continuum is less heavily absorbed and dilutes the continuum polarization. The scattered flux is also less absorbed in these lower opacity regions of the wind such that for BAL QSOs with detached absorptions, lower continuum polarization and shallow troughs in the polarized flux are observed.

However, with polarization data only, we cannot unambiguously locate the scatterers. A convenient way to solve this problem is to compare the polarization position angle with the orientation of the radio-axis. Unfortunately, only 3 BAL QSOs have been resolved at the radio wavelengths and have polarimetric data available. Interestingly, in these 3 objects, the optical polarization angle is nearly perpendicular to the radio-axis, as expected from a model where scattering occurs in a polar region.

Acknowledgements. H. Lamy thanks Prof. J. Lemaire and the Belgian Institute of Space Aeronomy for giving him the opportunity of performing observations in La Silla (Chile) in March 2002. The authors would also like to thank the referee for the careful reading. 


\section{References}

Angonin, M.-C., Vanderriest, C., Remy, M., et al. 1990, A\&A, 233, L5

Arav, N. 1997, in Accretion Phenomena and Related Outflows, ed. D. T. Wickramasingue et al., IAU Coll., 163, ASP Conf. Ser., 121,580

Baldwin, J. A., \& Stone, R. P. S. 1984, MNRAS, 204, 347

Becker, R. H., White, R. L., \& Helfand, D. J. 1995, ApJ, 450, 559

Becker, R. H., Gregg, M. D., Hook, I. M., et al. 1997, ApJ, 479, 93

Becker, R. H., White, R. L., Gregg, M. D., et al. 2000, ApJS, 538, 72

Becker, R. H., White, R. L., Gregg, M. D., et al. 2001, ApJ, 135, 227

Begelman, M., de Kool, M., \& Sikora, M. 1991, ApJ, 382, 416

Boroson, T. A., \& Green, R. F. 1992, ApJS, 80, 109

Boroson, T. A., \& Meyers, K. A. 1992, ApJ, 397, 442

Boroson, T. A. 2002, ApJ, 565, 78

Brandt, W. N., Comastri, A., Gallagher, S. C., et al. 1999, ApJ, 525, 69

Brotherton, M. S., Tran, H. D., van Breugel, W., et al. 1998, ApJ, 487, L113

Brotherton, M. S., van Breugel, W., Smith, R. J., et al. 1998, ApJ, 505, L7

Brotherton, M. S., Croom, S. M., De Breuck, C., et al. 2002, AJ, 124, 2575

Brown, J. C., \& Mc Lean, I. S. 1977, A\&A, 57, 141

Cassinelli, J. P., Nordsieck, K. H., \& Murison, M. A. 1987, ApJ, 317, 290

Chaffee, F. H., Foltz, C. B., Hewett, P. C., et al. 1991, AJ, 102, 1627

Chiang, J., \& Murray, N. 1996, ApJ, 466, 704

Clavel, J., Reichert, G. A., Alloin, D., et al. 1991, ApJ, 366, 64

Clarke, D., \& Stewart, B. G. 1986, VA, 29, 27

Cohen, M. H., Ogle, P. M., Tran, H. D., et al. 1995, ApJ, 448, 77

de Kool, M., \& Begelman, M. 1995, ApJ, 455, 448

di Serego Alighieri, S., Cimatti, A., \& Fosbury, R. A. E. 1994, ApJ, 431,123

di Serego Alighieri, S. 1998, in Instrumentation for Large Telescopes, ed. J. M. Rodriguez Espinosa (Cambridge University Press), 287

Everett, J. E. 2002 [arXiv: astro-ph/0212421]

Foltz, C. B., Chaffee, F. H., Hewett, P. C., et al. 1989, AJ, 98, 1959

Francis, P. J., \& Wills, B. J. 1999, in Quasars and cosmology, ed. G. Ferland et al., ASP Conf. Ser., 162, 363

Gallagher, S. C., Brandt, W. N., Sambruna, R. M., et al. 1999, ApJ, 519,549

Glenn, J., Schmidt, G. D., \& Foltz, C. B. 1994, ApJ, 434, 47

Goodrich, R. W., \& Miller, J. S. 1995, ApJ, 448, 73

Goodrich, R. W. 1997, ApJ, 474, 606

Green, P. J., Aldcroft, T. L., Mathur, S., et al. 2001, ApJ, 558, 109

Hall, P. B., Anderson, S. F., Strauss, M. A., et al. 2002, ApJS, 141, 267

Hartig, G. F., \& Baldwin, J. A. 1986, ApJ, 302, 64

Hazard, C., McMahon, R., Webb, J. K., \& Morton, D. C. 1987, ApJ, 323,263

Hewett, P. C., Foltz, C. B., Chaffee, F. H., et al. 1991, AJ, 101, 1121

Hewett, P. C., \& Foltz, C. B. 2003, AJ, 125, 1784

Hines, D. C., \& Wills, B. J. 1995, ApJ, 448, 69

Hutsemékers, D. 1993, A\&A, 280, 435

Hutsemékers, D., Lamy, H., \& Remy, M. 1998, A\&A, 340, 371 (Paper I)

Hutsemékers, D., \& Lamy, H. 2000, A\&A, 358, 835 (Paper II)

Hutsemékers, D., \& Lamy, H. 2002, in Mass outflows in Active Galactic Nuclei: new perspectives, ed. Crenshaw et al., ASP Conf. Ser., 255, 207

Hutsemékers, D., Sluse, D., Cabanac, R., et al. 2004, A\&A, in preparation
Jiang, D. R., \& Wang, T. G. 2003, A\&A, 397, L13

Kellermann, K. I., Doeleman, S., Wright, M. C. H., et al. 1994, AJ, 108,1163

Korista, K. T., Voit, G. M., Morris, S. L., \& Weymann, R. J. 1993, ApJS, 88, 357

Kuncic, Z. 1999, PASP, 111, 954

Lamy, H., \& Hutsemékers, D. 1999, in Structure and kinematics of the Broad Line Region, ed. M. Gaskell, ASP Conf. Ser., 175, 125

Lamy, H., \& Hutsemékers, D. 2000a, A\&AS, 142, 451

Lamy, H., \& Hutsemékers, D. 2000b, A\&A, 356, L9

Lamy, H. 2003, Ph.D. Thesis: Contribution à l'étude de la polarisation des quasars de type BAL, University of Liège

Lee, H. W. 1994, MNRAS, 268, 49

Lee, H. W., \& Blandford, R. D. 1997, MNRAS, 288, 19

Low, F.J., Cutri, R. M., Kleinmann, S. G., et al. 1989, ApJ, 340, 1

Magain, P., Surdej, J., Swings, J.-P., et al. 1988, Nature, 334, 325

Melnick, J., Dekker, H., \& D'odorico, S. 1989, EFOSC, ESO Operating Manual 4, Version 2, ESO

Menou, K., Vanden Berk, D. E., Ivezic, Z., et al. 2001, ApJ, 561, 645

Moore, R. L., \& Stockman, H. S. 1984, ApJ, 279, 465

Murray, N., Chiang, J., Grossman, S. A., \& Voit, G. M. 1995, ApJ, 451, 498

Murtagh, F., \& Heck, A. 1987, Multivariate Data Analysis (D. Reidel Publishing Company)

Ogle, P. M. 1997 in Mass Ejection from Active Galactic Nuclei, ed. N. Arav et al., ASP Conf. Ser., 128, 78

Ogle, P. M., Cohen, M. H., Miller, J. S., et al. 1999, ApJS, 125, 1

Østensen, R., Remy, M., Lindblad, P. O., et al. 1997, A\&AS, 126, 393

Pereyra, N. A., Owocki, S. P., Hillier, D. J., et al. 2004, ApJ, 608, 454

Press, W. H., Flannery, B. P., Teukolsky, S. A., \& Vetterling, W. T. 1989, Numerical Recipes (Cambdridge University Press)

Proga, D., Stone, J. M., \& Drew, J. E. 1998, MNRAS, 295, 595

Proga, D., Stone, J. M., \& Drew, J. E. 1999, MNRAS, 310, 476

Proga, D. 2000, ApJ, 543, 686

Proga, D. 2003, ApJ, 585, 406

Punsly, B. 1999, ApJ, 527, 624

Reichard, T. A., Richards, G. T., Schneider, D. P., et al. 2003, AJ, 125 , 1711

Schmidt, G. D., \& Hines, D. C. 1997, in Mass Ejection from Active Galactic Nuclei, ed. N. Arav et al., ASP Conf. Ser., 128, 106

Schmidt, G. D., \& Hines, D. C. 1999, ApJ, 512, 125

Scoville, N., \& Norman, C. 1995, ApJ, 451, 510

Serkowski, K., Matthewson, D. S., \& Ford, V. L. 1975, ApJ, 196, 261

Simmons, J. F. L., \& Stewart, B. G. 1985, A\&A, 142, 100

Sluse, D., Hutsemékers, D., Lamy, H., \& Cabanac, R. 2004, A\&A, in preparation

Sprayberry, D., \& Foltz, C. B. 1992, ApJ, 390, 39

Stockman, H. S., Hier, R. G., \& Angel, J. R. P. 1981, ApJ, 243, 404

Stone, R. P. S., \& Baldwin, J. A. 1983, MNRAS, 204, 347

Stoughton, C., Lupton, R. H., Bernardi, M., et al. 2002, AJ, 123, 485

Surdej, J., \& Hutsemékers, D. 1987, A\&A, 177, 42

Tolea, A., Krolik, J. H., \& Tsvetanov, Z. 2002, ApJ, 578, L31

Turnshek, D. A. 1988, in QSO absorption lines: probing the Universe, ed. J. C. Blades et al. (Cambridge), 17

Véron-Cetty, M. P., \& Véron, P. 2000, A catalogue of quasars and active nuclei, 9th ed. Garching: European Southern Observatory (ESO), ESO Scientific Report No. 19

Voit, G. M., Weymann, R. J., \& Korista, K. T. 1993, ApJ, 413, 95

Wardle, J. F. C., \& Kronberg, P. P. 1974, ApJ, 194, 249

Weymann, R. J., Morris, S. L., Foltz, C. B., \& Hewett, P. C. 1991, ApJ, 373, 23

Yamamoto, T. M., \& Vansevicius, V. 1999, PASJ, 51, 405

Yuan, M. J., \& Wills, B. J. 2003, ApJ, 593, 11 